\title{
Dynamics of quantum phases in a spinor condensate
}

\author{
S. Yi, ${ }^{1}$ Ö. E. Müstecaplıoğlu, ${ }^{1,2}$ and L. You ${ }^{1}$ \\ ${ }^{1}$ School of Physics, Georgia Institute of Technology, Atlanta, Georgia 30332, USA \\ ${ }^{2}$ Koc University, Rumelifeneri Yolu, 34450 Sariyer, Istanbul, Turkey
}

(Received 22 February 2003; published 30 July 2003)

\begin{abstract}
We discuss the quantum phases and their diffusion dynamics in a spinor-1 atomic Bose-Einstein condensate. For ferromagnetic interactions, we obtain the exact ground-state distribution of the phases associated with the total atom number $(N)$, the total magnetization $(\mathcal{M})$, and the alignment (or hypercharge) $(Y)$ of the system. The mean-field ground state is stable against fluctuations of atom numbers in each of the spin components, and the phases associated with the order parameter for each spin components diffuse while dynamically recovering the two broken continuous symmetries [U(1) and $\mathrm{SO}(2)]$ when $N$ and $\mathcal{M}$ are conserved, as in current experiments. We discuss the implications to the quantum dynamics due to an external (homogeneous) magnetic field. We also comment on the case of a spinor-1 condensate with antiferromagnetic interactions.
\end{abstract}

DOI: 10.1103/PhysRevA.68.013613

PACS number(s): 03.75.Mn, 03.75.Kk

\section{INTRODUCTION}

Since the observation of Bose-Einstein condensation in trapped dilute alkali atoms [1-3], the coherence properties of a condensate have become the focus of many theoretical studies [4-9]. As it was initially pointed out in Refs. [4,5], the number fluctuations associated with the assumption of a coherent-state macroscopic condensate wave function lead to the "diffusion" (or spreading) of the initial phase. For a scalar, one-component atomic condensate, this diffusion, the attempt to restore the $\mathrm{U}(1)$ symmetry of the underline interacting system, can be formulated in terms of the dynamics of a zero mode, or the Goldstone mode [4]. More physically meaningful discussions, in terms of the relative phase of the two condensates, were carried out soon afterwards [6-9]. Starting with the remarkable direct observation of the firstorder coherence in an interference experiment [10], direct relationship between the number fluctuation and quantum phase dynamics was first observed with a condensate in a periodic potential [11], and more recently, in the remarkable Mott insulating state $[12,13]$.

The emergence of spinor condensates [14,15] (of atoms with hyperfine quantum number $F=1$ ) has created new opportunities to understand quantum coherence and the associated phase dynamics within a three-component condensate $[16,17]$. In an earlier paper [18], we have presented important physical insight into quantum phase diffusions of a spinor condensate. We have investigated the coupled zero mode dynamics as a consequence of the usual mean-field treatment that calls for the breaking of two continuous symmetries: a $\mathrm{U}(1)$ gauge transformation $e^{i \theta}$ and $\mathrm{SO}(3)$ spin rotations $\mathcal{U}(\alpha, \beta, \tau)=e^{-i F_{z} \alpha} e^{-i F_{y} \beta} e^{-i F_{z} \tau}$ (in the absence of an external magnetic field) [19].

This paper provides a detailed investigation of the quantum phase dynamics for a spinor- 1 condensate. As before, we will focus on the case of ferromagnetic interactions, for which the validity of the single spatial mode approximation proves to be a convenient starting point for the emergence of transparent physical illustrations of the zero mode dynamics [20]. The fluctuations of atom numbers in each of the spin states will be shown to be connected directly, in this case, to the fluctuations of the pointing direction of the macroscopic spin (or magnetic dipole moment) of the condensate. This paper is organized as follows. In Sec. II, after introducing the Hamiltonian for a spinor-1 condensate, we explicitly work out the Heisenberg equations for the atomic field operators. Following the standard Bogoliubov approach [21], we obtain the coupled Gross-Pitaevskii equations for the mean field of the condensate and the coupled Bogoliubov-de Gennes equations for the quantum fluctuations. We then generalize the Hermitian operators for the condensate number and phase fluctuations (of the Goldstone mode), as introduced earlier [4], to each of the spin components, and derive their dynamic equations under the rotating wave-function approximation. In Sec. III, we perform a detailed study of the stationary phase diffusion of a spinor-1 condensate within the single mode approximation (SMA) valid exactly for ferromagnetic interactions. Section IV addresses the influence of an external magnetic field and in Sec. V we discuss the same phase dynamics for a condensate with antiferromagnetic interactions. We conclude in Sec. VI.

\section{FORMULATION}

We consider a system of $N$ spin-1 bosonic atoms interacting via only $s$-wave scattering $[19,22-24]$. In the secondquantized form, the Hamiltonian of our system becomes $[19,22]$

$$
\begin{aligned}
H= & \sum_{i} \int d \vec{r} \psi_{i}^{\dagger}(\vec{r})\left[-\frac{\hbar^{2} \nabla^{2}}{2 M}+V_{\mathrm{ext}}(\vec{r})\right] \psi_{i}(\vec{r}) \\
& +\frac{c_{0}}{2} \sum_{i, j} \int d \vec{r} \psi_{i}^{\dagger}(\vec{r}) \psi_{j}^{\dagger}(\vec{r}) \psi_{i}(\vec{r}) \psi_{j}(\vec{r}) \\
& +\frac{c_{2}}{2} \sum_{i, j, k, l} \int d \vec{r} \psi_{i}^{\dagger}(\vec{r}) \psi_{j}^{\dagger}(\vec{r}) \overrightarrow{\mathbf{F}}_{i k} \cdot \overrightarrow{\mathbf{F}}_{j l} \psi_{l}(\vec{r}) \psi_{k}(\vec{r}),
\end{aligned}
$$

where $\psi_{j}(\vec{r})(j=+, 0,-)$ denotes the annihilation operator for the $j$ th component of a spinor-1 field. The external trapping potential $V_{\text {ext }}(\vec{r})$ is assumed to be spin independent, as in a far off-resonant optical dipole force trap so that atomic 
spin degrees of freedom are completely accessible. The pair interaction coefficients are $c_{0}=4 \pi \hbar^{2}\left(a_{0}+2 a_{2}\right) / 3 M$ and $c_{2}$ $=4 \pi \hbar^{2}\left(a_{2}-a_{0}\right) / 3 M$, with $a_{0}\left(a_{2}\right)$ being the $s$-wave scattering length for two spin-1 atoms in the combined symmetric channel of total spin 0 (2), and $M$ is the mass of atom. $\overrightarrow{\mathbf{F}}$ is spin-1 matrix representation. The interacting terms can be regrouped to show that they include, respectively, selfscattering, cross scattering, and the spin relaxation [24].

From Hamiltonian (1), one can easily derive the Heisenberg equations for field operators

$$
\begin{aligned}
i \hbar \dot{\psi}_{+}= & {\left[-\frac{\hbar^{2} \nabla^{2}}{2 M}+V_{\mathrm{ext}}(\vec{r})\right] \psi_{+}+c_{2} \psi_{-}^{\dagger} \psi_{0}^{2}+c_{0}\left(\psi_{+}^{\dagger} \psi_{+}\right.} \\
& \left.+\psi_{0}^{\dagger} \psi_{0}+\psi_{-}^{\dagger} \psi_{-}\right) \psi_{+}+c_{2}\left(\psi_{+}^{\dagger} \psi_{+}+\psi_{0}^{\dagger} \psi_{0}\right. \\
& \left.-\psi_{-}^{\dagger} \psi_{-}\right) \psi_{+}, \\
i \hbar \dot{\psi}_{0}= & \left.-\frac{\hbar^{2} \nabla^{2}}{2 M}+V_{\mathrm{ext}}(\vec{r})\right] \psi_{0}+2 c_{2} \psi_{0}^{\dagger} \psi_{+} \psi_{-}+c_{0}\left(\psi_{+}^{\dagger} \psi_{+}\right. \\
+ & \left.\psi_{0}^{\dagger} \psi_{0}+\psi_{-}^{\dagger} \psi_{-}\right) \psi_{0}+c_{2}\left(\psi_{+}^{\dagger} \psi_{+}+\psi_{-}^{\dagger} \psi_{-}\right) \psi_{0}, \\
i \hbar \dot{\psi}_{-}= & {\left[-\frac{\hbar^{2} \nabla^{2}}{2 M}+V_{\mathrm{ext}}(\vec{r})\right] \psi_{-}+c_{2} \psi_{+}^{\dagger} \psi_{0}^{2}+c_{0}\left(\psi_{+}^{\dagger} \psi_{+}\right.} \\
& \left.+\psi_{0}^{\dagger} \psi_{0}+\psi_{-}^{\dagger} \psi_{-}\right) \psi_{-}+c_{2}\left(\psi_{-}^{\dagger} \psi_{-}+\psi_{0}^{\dagger} \psi_{0}\right. \\
& \left.-\psi_{+}^{\dagger} \psi_{+}\right) \psi_{-} .
\end{aligned}
$$

Following the Hartree mean-field theory, we assume that there are three "large" condensate components $\phi_{j}$ around which we can study the small quantum fluctuations (offcondensate excitations) via the Bogoluibov approximation

$$
\psi_{j}(\vec{r})=\sqrt{N} \phi_{j}(\vec{r})+\delta \psi_{j}(\vec{r})
$$

with $N$ being the total number of atoms. We note that such an approach can become questionable in a multicomponent system where a certain component becomes negligibly small and its quantum nature becomes important. For ferromagnetic interactions, however, our previous studies [20] show that the ground-state wave functions can always be expressed as $\phi_{j} \propto \phi$ and hence the Bogoluibov prescription can always be applied. By substituting Eq. (3) into Eq. (2), we find, in the zeroth order, the coupled Gross-Pitaevskii equations (GPEs)

$$
\begin{aligned}
& i \hbar \dot{\phi}_{+}=\left[\mathcal{L}+c_{0} \rho+c_{2}\left(\rho_{+}+\rho_{0}-\rho_{-}\right)\right] \phi_{+}+c_{2} N \phi_{-}^{*} \phi_{0}^{2}, \\
& i \hbar \dot{\phi}_{0}=\left[\mathcal{L}+c_{0} \rho+c_{2}\left(\rho_{+}+\rho_{-}\right)\right] \phi_{0}+2 c_{2} N \phi_{0}^{*} \phi_{+} \phi_{-}, \\
& i \hbar \dot{\phi}_{-}=\left[\mathcal{L}+c_{0} \rho+c_{2}\left(\rho_{-}+\rho_{0}-\rho_{+}\right)\right] \phi_{-}+c_{2} N \phi_{+}^{*} \phi_{0}^{2},
\end{aligned}
$$

with $\mathcal{L}=-\hbar^{2} \nabla^{2} / 2 M+V_{\text {ext }}(\vec{r}), j$ th component condensate density $\rho_{j}(\vec{r})=N\left|\phi_{j}(\vec{r})\right|^{2}$, and total condensate density $\rho(\vec{r})=\rho_{+}(\vec{r})+\rho_{0}(\vec{r})+\rho_{-}(\vec{r}) . \int d \vec{r} \rho_{j}(\vec{r})=N_{j}$ is the number of condensed atoms in the $j$ th component. In the first order, the quantum fluctuations obey the following set of Bogoluibov-de Gennes equations (BdGEs):

$$
\begin{aligned}
i \hbar \frac{\partial \delta \psi_{+}}{\partial t}= & {\left[\mathcal{L}+c_{0}\left(\rho+\rho_{+}\right)+c_{2}\left(2 \rho_{+}+\rho_{0}-\rho_{-}\right)\right] \delta \psi_{+} } \\
& +N\left(c_{+} \phi_{0}^{*} \phi_{+}+2 c_{2} \phi_{-}^{*} \phi_{0}\right) \delta \psi_{0} \\
& +c_{-} N \phi_{-}^{*} \phi_{+} \delta \psi_{-}+c_{+} N \phi_{+}^{2} \delta \psi_{+}^{\dagger} \\
& +c_{+} N \phi_{+} \phi_{0} \delta \psi_{0}^{\dagger}+N\left(c_{-} \phi_{+} \phi_{-}+c_{2} \phi_{0}^{2}\right) \delta \psi_{-}^{\dagger}, \\
i \hbar \frac{\partial \delta \psi_{0}}{\partial t}=[ & \left.+c_{0}\left(\rho+\rho_{0}\right)+c_{2}\left(\rho_{+}+\rho_{-}\right)\right] \delta \psi_{0}+N\left(c_{+} \phi_{+}^{*} \phi_{0}\right. \\
+ & \left.2 c_{2} \phi_{0}^{*} \phi_{-}\right) \delta \psi_{+}+N\left(c_{+} \phi_{-}^{*} \phi_{0}\right. \\
+ & \left.2 c_{2} \phi_{0}^{*} \phi_{+}\right) \delta \psi_{-}+c_{+} N \phi_{+} \phi_{0} \delta \psi_{+}^{\dagger} \\
+ & c_{+} N \phi_{0} \phi_{-} \delta \psi_{-}^{\dagger}+N\left(c_{0} \phi_{0}^{2}+2 c_{2} \phi_{+} \phi \phi_{-}\right) \delta \psi_{0}^{\dagger}, \\
& +c_{+} N \phi_{-} \phi_{0} \delta \psi_{0}^{\dagger}+N\left(c_{-} \phi_{+} \phi_{-}+c_{2} \phi_{0}^{2}\right) \delta \psi_{+}^{\dagger}, \\
& +c_{-} N \phi_{+}^{*} \phi_{-} \delta \psi_{+}+c_{+} N \phi_{-}^{2} \delta \psi_{-}^{\dagger} \\
& +N\left(c_{+} \phi_{0}^{*} \phi_{-}+2 c_{2} \phi_{+}^{*} \phi_{0}\right) \delta \psi_{0} \\
= & {\left[\mathcal{L}+c_{0}\left(\rho+\rho_{-}\right)+c_{2}\left(2 \rho_{-}+\rho_{0}-\rho_{+}\right)\right] \delta \psi_{-} } \\
& \\
& \\
& \\
& \\
&
\end{aligned}
$$

where we have defined $c_{ \pm} \equiv c_{0} \pm c_{2}$.

To study the quantum phase dynamics, we introduce number fluctuation operators [4]

$$
P_{j}=\int d \vec{r}\left[\phi_{j}^{*}(\vec{r}) \delta \psi_{j}(\vec{r})+\phi_{j}(\vec{r}) \delta \psi_{j}^{\dagger}(\vec{r})\right]
$$

Using GPEs (4) and BdGEs (5) above, we find the following evolution equations for these operators:

$$
\begin{aligned}
i \hbar \frac{\partial P_{+}}{\partial t}= & -\frac{i \hbar}{2} \frac{\partial P_{0}}{\partial t}=i \hbar \frac{\partial P_{-}}{\partial t}=c_{2} N \int d \vec{r}\left(\phi_{-}^{*} \phi_{0}^{2} \delta \psi_{+}^{\dagger}\right. \\
& \left.+\phi_{+}^{*} \phi_{0}^{2} \delta \psi_{-}^{\dagger}+2 \phi_{+}^{*} \phi_{-}^{*} \phi_{0} \delta \psi_{0}-\text { H.c. }\right)
\end{aligned}
$$


Thus, both $P_{\text {tot }}=P_{+}+P_{0}+P_{-}$and $P_{+}-P_{-}$are constants of motion, which is, in fact, obvious since Hamiltonian (1) conserves the total number of atoms and the total magnetization.

We define conjugate phase operator $Q_{\text {tot }}=Q_{+}+Q_{0}+Q_{-}$ according to

$$
Q_{j}=i \hbar \int d \vec{r}\left[\theta_{j}^{*}(\vec{r}) \delta \psi_{j}(\vec{r})-\theta_{j}(\vec{r}) \delta \psi_{j}^{\dagger}(\vec{r})\right]
$$

where $\theta_{j}(\vec{r})$ are the phase functions to be determined [4]. Canonical quantization condition $\left[Q_{\text {tot }}, P_{\text {tot }}\right]=i \hbar$ is satisfied if constraint $J_{+}+J_{0}+J_{-} \equiv 1$ is enforced with $J_{j}$ $\equiv \int d \vec{r}\left[\theta_{j}^{*}(\vec{r}) \phi_{j}(\vec{r})+\theta_{j}(\vec{r}) \phi_{j}^{*}(\vec{r})\right]$. From the defining equation for $Q_{\text {tot }}$,

$$
\frac{d Q_{\text {tot }}}{d t}=N \tilde{u} P_{\text {tot }},
$$

the Goldstone mode inertial parameter $\tilde{u}$ can be determined. We obtain the dynamic equations for the phase functions as

$$
\begin{aligned}
i \hbar \dot{\theta}_{+}= & {\left[\mathcal{L}+c_{0}\left(\rho+\rho_{+}\right)+c_{2}\left(2 \rho_{+}+\rho_{0}-\rho_{-}\right)\right] \theta_{+} } \\
& +N\left(c_{+} \phi_{0}^{*} \phi_{+}+2 c_{2} \phi_{-}^{*} \phi_{0}\right) \theta_{0}+c_{-} N \phi_{-}^{*} \phi_{+} \theta_{-} \\
& +c_{+} N \phi_{+}^{2} \theta_{+}^{*}+c_{+} N \phi_{+} \phi_{0} \theta_{0}^{*}+N\left(c_{-} \phi_{+} \phi_{-}\right. \\
& \left.+c_{2} \phi_{0}^{2}\right) \theta_{-}^{*}-N \tilde{u} \phi_{+}, \\
i \hbar \dot{\theta}_{0}= & {\left[\mathcal{L}+c_{0}\left(\rho+\rho_{0}\right)+c_{2}\left(\rho_{+}+\rho_{-}\right)\right] \theta_{0}+N\left(c_{+} \phi_{+}^{*} \phi_{0}\right.} \\
& \left.+2 c_{2} \phi_{0}^{*} \phi_{-}\right) \theta_{+}+N\left(c_{+} \phi_{-}^{*} \phi_{0}+2 c_{2} \phi_{0}^{*} \phi_{+}\right) \theta_{-} \\
& +c_{+} N \phi_{+} \phi_{0} \theta_{+}^{*}+c_{+} N \phi_{0} \phi_{-} \theta_{-}^{*}+N\left(c_{0} \phi_{0}^{2}\right. \\
& \left.+2 c_{2} \phi_{+} \phi_{-}\right) \theta_{0}^{*}-N \tilde{u} \phi_{0}, \\
i \hbar \dot{\theta}_{-}= & {\left[\mathcal{L}+c_{0}\left(\rho+\rho_{-}\right)+c_{2}\left(2 \rho_{-}+\rho_{0}-\rho_{+}\right)\right] \theta_{-} } \\
& +N\left(c_{+} \phi_{0}^{*} \phi_{-}+2 c_{2} \phi_{+}^{*} \phi_{0}\right) \theta_{0}+c_{-} N \phi_{+}^{*} \phi_{-} \theta_{+} \\
& +c_{+} N \phi_{-}^{2} \theta_{-}^{*}+c_{+} N \phi_{-} \phi_{0} \theta_{0}^{*}+N\left(c_{-} \phi_{+} \phi_{-}\right. \\
& \left.+c_{2} \phi_{0}^{2}\right) \theta_{+}^{*}-N \tilde{u} \phi_{-} .
\end{aligned}
$$

Combining Eqs. (5) and (10), the dynamic equations of $Q_{j}$ can be easily obtained to be

$$
\begin{aligned}
\dot{Q}_{+}= & N \tilde{u} P_{+}+N \int d \vec{r}\left\{-\left[\left(c_{+} \phi_{+}^{*} \phi_{0}+2 c_{2} \phi_{0}^{*} \phi_{-}\right) \theta_{0}^{*}+c_{+} \phi_{+}^{*} \phi_{0}^{*} \theta_{0}+\left(c_{-} \phi_{+}^{*} \phi_{-}^{*}+c_{2} \phi_{0}^{* 2}\right) \theta_{-}+c_{-} \phi_{+}^{*} \phi_{-} \theta_{-}^{*}\right] \delta \psi_{+}\right. \\
& \left.+\left(c_{+} \phi_{0}^{*} \phi_{+} \theta_{+}^{*}+c_{+} \phi_{+}^{*} \phi_{0}^{*} \theta_{+}+2 c_{2} \phi_{-}^{*} \phi_{0} \theta_{+}^{*}\right) \delta \psi_{0}+\left(c_{-} \phi_{+}^{*} \phi_{-}^{*} \theta_{+}+c_{-} \phi_{-}^{*} \phi_{+} \theta_{+}^{*}+c_{2} \phi_{0}^{* 2} \theta_{+}\right) \delta \psi_{-}+\text {H.c. }\right\}, \\
\dot{Q}_{0}= & N \tilde{u} P_{0}+N \int d \vec{r}\left\{-\left[\left(c_{+} \phi_{0}^{*} \phi_{+}+2 c_{2} \phi_{-}^{*} \phi_{0}\right) \theta_{+}^{*}+c_{+} \phi_{+}^{*} \phi_{0}^{*} \theta_{+}+\left(c_{+} \phi_{0}^{*} \phi_{-}+2 c_{2} \phi_{+}^{*} \phi_{0}\right) \theta_{-}^{*}+c_{+} \phi_{0}^{*} \phi_{-}^{*} \theta_{-}\right] \delta \psi_{0}\right. \\
+ & \left.\left(c_{+} \phi_{+}^{*} \phi_{0} \theta_{0}^{*}+c_{+} \phi_{+}^{*} \phi_{0}^{*} \theta_{0}+2 c_{2} \phi_{0}^{*} \phi_{-} \theta_{0}^{*}\right) \delta \psi_{+}+\left(c_{+} \phi_{-}^{*} \phi_{0} \theta_{0}^{*}+c_{+} \phi_{0}^{*} \phi_{-}^{*} \theta_{0}+2 c_{2} \phi_{0}^{*} \phi_{+} \theta_{0}^{*}\right) \delta \psi_{-}+\text {H.c. }\right\}, \\
\dot{Q}_{-}= & N \tilde{u} P_{-}+N \int d \vec{r}\left\{-\left[\left(c_{+} \phi_{-}^{*} \phi_{0}+2 c_{2} \phi_{0}^{*} \phi_{+}\right) \theta_{0}^{*}+c_{+} \phi_{-}^{*} \phi_{0}^{*} \theta_{0}+\left(c_{-} \phi_{+}^{*} \phi_{-}^{*}+c_{2} \phi_{0}^{* 2}\right) \theta_{+}+c_{-} \phi_{-}^{*} \phi_{+} \theta_{+}^{*}\right] \delta \psi_{-}\right. \\
& \left.+\left(c_{+} \phi_{0}^{*} \phi_{-} \theta_{-}^{*}+c_{+} \phi_{-}^{*} \phi_{0}^{*} \theta_{-}+2 c_{2} \phi_{+}^{*} \phi_{0} \theta_{-}^{*}\right) \delta \psi_{0}+\left(c_{-} \phi_{+}^{*} \phi_{-}^{*} \theta_{-}+c_{-} \phi_{+}^{*} \phi_{-} \theta_{-}^{*}+c_{2} \phi_{0}^{* 2} \theta_{-}\right) \delta \psi_{+}+\text {H.c. }\right\} .
\end{aligned}
$$

We note that $\left[Q_{j}, P_{k}\right]=i \hbar \delta_{j k} J_{j},\left[\delta \psi_{j}(\vec{r}), P_{k}\right]=\delta_{j k} \phi_{j}(\vec{r})$, and $\left[\delta \psi_{j}(\vec{r}), Q_{k}\right]=-i \hbar \delta_{j k} \theta_{j}(\vec{r})$. This prompts us to make the rotating wave approximation (RWA) [7,9] by assuming that $\delta \psi_{j}(\vec{r})=\sum_{j} a_{j}(\vec{r}) P_{j}+\sum_{j} b_{j}(\vec{r}) Q_{j}$, which leads to $a_{j}(\vec{r})$ $=\theta_{j}(\vec{r}) / J_{j}$ and $b_{j}(\vec{r})=\phi_{j}(\vec{r}) / i \hbar J_{j}$. Therefore, under the RWA, we obtain $\delta \psi_{j}(\vec{r})=\theta_{j}(\vec{r}) P_{j} / J_{j}+\phi_{j}(\vec{r}) Q_{j} / i \hbar J_{j}$. With this result, the complete dynamic equations for the number and phase fluctuation operators become 


$$
\begin{aligned}
& \dot{P}_{+}=-\frac{\dot{P}_{0}}{2} \\
& =\dot{P}_{-}=\frac{2 c_{2} N}{\hbar}\left[\frac{\left(\phi_{-} \theta_{+} \mid \phi_{0}^{2}\right)^{\prime \prime}}{J_{+}} P_{+}-2 \frac{\left(\phi_{0} \theta_{0} \mid \phi_{+} \phi_{-}\right)^{\prime \prime}}{J_{0}} P_{0}+\frac{\left(\phi_{+} \theta_{-} \mid \phi_{0}^{2}\right)^{\prime \prime}}{J_{-}} P_{-}\right]+\frac{2 c_{2} N}{\hbar^{2}}\left(\phi_{+} \phi_{-} \mid \phi_{0}^{2}\right)^{\prime}\left(\frac{Q_{+}}{J_{+}}-\frac{2 Q_{0}}{J_{0}}+\frac{Q_{-}}{J_{-}}\right), \\
& \dot{Q}_{+}=N \tilde{u} P_{+}-\frac{2 N}{J_{+}}\left[2 c_{-} I_{-+}+2 c_{+} I_{+0}+2 c_{2}\left(\phi_{0} \theta_{0} \mid \phi_{-} \theta_{+}\right)^{\prime}+c_{2}\left(\phi_{0}^{2} \mid \theta_{-} \theta_{+}\right)^{\prime}\right] P_{+}+\frac{4 N}{J_{0}}\left[c_{+} I_{+0}+c_{2}\left(\phi_{0} \theta_{0} \mid \phi_{-} \theta_{+}\right)^{\prime}\right] P_{0} \\
& +\frac{2 N}{J_{-}}\left[2 c_{-} I_{-+}+c_{2}\left(\phi_{0}^{2} \mid \theta_{+} \theta_{-}\right)^{\prime}\right] P_{-}+\frac{2 c_{2} N}{\hbar J_{+}}\left[2\left(\phi_{+} \phi_{-} \mid \phi_{0} \theta_{0}\right)^{\prime \prime}+\left(\phi_{+} \theta_{-} \mid \phi_{0}^{2}\right)^{\prime \prime}\right] Q_{+}+\frac{4 c_{2} N}{\hbar J_{0}}\left(\phi_{-} \theta_{+} \mid \phi_{0}^{2}\right)^{\prime \prime} Q_{0} \\
& +\frac{2 c_{2} N}{\hbar J_{-}}\left(\phi_{0}^{2} \mid \theta_{+} \phi_{-}\right)^{\prime \prime} Q_{-}, \\
& \dot{Q}_{0}=N \tilde{u} P_{0}-\frac{4 N}{J_{0}}\left[c_{+} I_{+0}+c_{+} I_{-0}+c_{2}\left(\phi_{-} \theta_{+} \mid \phi_{0} \theta_{0}\right)^{\prime}+c_{2}\left(\phi_{+} \theta_{-} \mid \phi_{0} \theta_{0}\right)^{\prime}\right] P_{0}+\frac{4 N}{J_{+}}\left[c_{+} I_{+0}+c_{2}\left(\phi_{0} \theta_{0} \mid \phi_{-} \theta_{+}\right)^{\prime}\right] P_{+} \\
& +\frac{4 N}{J_{-}}\left[c_{+} I_{-0}+c_{2}\left(\phi_{0} \theta_{0} \mid \phi_{+} \theta_{-}\right)^{\prime}\right] P_{-}+\frac{4 c_{2} N}{\hbar J_{+}}\left(\phi_{0} \theta_{0} \mid \phi_{+} \phi_{-}\right)^{\prime \prime} Q_{+}+\frac{4 c_{2} N}{\hbar J_{0}}\left[\left(\phi_{0}^{2} \mid \phi_{-} \theta_{+}\right)^{\prime \prime}+\left(\phi_{0}^{2} \mid \phi_{+} \theta_{-}\right)^{\prime \prime}\right] Q_{0} \\
& +\frac{4 c_{2} N}{\hbar J_{-}}\left(\phi_{0} \theta_{0} \mid \phi_{+} \phi_{-}\right)^{\prime \prime} Q_{-}, \\
& \dot{Q}_{-}=N \tilde{u} P_{-}-\frac{2 N}{J_{-}}\left[2 c_{-} I_{-+}+2 c_{+} I_{-0}+2 c_{2}\left(\phi_{0} \theta_{0} \mid \phi_{+} \theta_{-}\right)^{\prime}+c_{2}\left(\phi_{0}^{2} \mid \theta_{+} \theta_{-}\right)^{\prime}\right] P_{-}+\frac{4 N}{J_{0}}\left[c_{+} I_{-0}+c_{2}\left(\phi_{0} \theta_{0} \mid \phi_{+} \theta_{-}\right)^{\prime}\right] P_{0} \\
& +\frac{2 N}{J_{+}}\left[2 c_{-} I_{-+}+c_{2}\left(\phi_{0}^{2} \mid \theta_{-} \theta_{+}\right)^{\prime}\right] P_{+}+\frac{2 c_{2} N}{\hbar J_{+}}\left(\phi_{0}^{2} \mid \theta_{-} \phi_{+}\right)^{\prime \prime} Q_{+}+\frac{4 c_{2} N}{\hbar J_{0}}\left(\phi_{+} \theta_{-} \mid \phi_{0}^{2}\right)^{\prime \prime} Q_{0}+\frac{2 c_{2} N}{\hbar J_{-}}\left[2\left(\phi_{-} \phi_{+} \mid \phi_{0} \theta_{0}\right)^{\prime \prime}\right. \\
& \left.+\left(\phi_{-} \theta_{+} \mid \phi_{0}^{2}\right)^{\prime \prime}\right] Q_{-},
\end{aligned}
$$

where we have introduced the following shorthand notation: $\quad(f g \mid h y)=\int d \vec{r} f^{*}(\vec{r}) g^{*}(\vec{r}) h(\vec{r}) y(\vec{r}), \quad(\cdot)^{\prime}=\operatorname{Re}(\cdot)$, $(\cdot)^{\prime \prime}=\operatorname{Im}(\cdot)$, and $I_{m l}=\int d \vec{r}\left(\phi_{m} \theta_{m}^{*}\right)^{\prime}\left(\phi_{l} \theta_{l}^{*}\right)^{\prime}$. Equation (12) thus completely determines the zero mode condensate fluctuations. Before finding the corresponding compact zero mode Hamiltonian in quadratic forms of $P_{j}$ and $Q_{j}$, we will first attempt to simplify Eq. (12), in the following section, using general properties of $\phi_{j}(\vec{r})$ and $\theta_{j}(\vec{r})$ for a ferromagnetic spinor-1 condensate [20].

\section{PHASE DIFFUSIONS AND THE ZERO MODES}

In general, the ground-state wave functions of a spinor condensate can be expressed as

$$
\phi_{j}(\vec{r}, t)=\left|\phi_{j}(\vec{r})\right| e^{-i \mu t / \hbar+i \alpha_{j}},
$$

with a common chemical potential $\mu$ for all spin components when the external magnetic field is negligible. Based on the minimization for the total energy Eq. (1) within the meanfield theory, it was shown in Refs. $[25,26]$ that there exists an important relation among $\alpha_{j}$, given by $\alpha_{+}+\alpha_{-}-2 \alpha_{0}=0$. We further proved in Ref. [20] that the ground-state wave function for each of the spin component takes the same spatial shape for (ferromagnetic interactions), i.e.,

$$
\phi_{j}(\vec{r})=\sqrt{n_{j}} \phi(\vec{r}) e^{i \alpha_{j}},
$$

with the real-valued mode function $\phi(\vec{r})$ (normalized to unity) governed by an equivalent scalar condensate GPE

$$
\left[\mathcal{L}+c_{+} N \phi^{2}(\vec{r})\right] \phi(\vec{r})=\mu \phi(\vec{r}),
$$

of a scattering length $a_{2}$ (note, $c_{+} \propto a_{2}$ ). Atoms, thus, only collide in the symmetric total spin $F=2$ channel in a ferromagnetic state in order to maintain their individual spins parallel. $n_{j}=N_{j} / N$ is the ratio of the number of atoms in the $i$ th spin component to the total number of atoms. For any given magnetization $\mathcal{M}, n_{j}$ is given explicitly by $n_{ \pm}=(1$ $\pm m)^{2} / 4$ and $n_{0}=\left(1-m^{2}\right) / 2$ with $m=\mathcal{M} / N$ [20,24].

Similarly, we look for phase functions of the form

$$
\theta_{j}(\vec{r}, t)=\left|\theta_{j}(\vec{r})\right| e^{-i \mu_{j} t / \hbar+i \alpha_{j}} .
$$

Substituting Eq. (16) into Eq. (10), we find that $\theta_{j}(\vec{r}, t)$ also evolve in time as $e^{-i \mu t / \hbar}$. To focus on the steady-state quantum fluctuation properties, we may, therefore, neglect the 


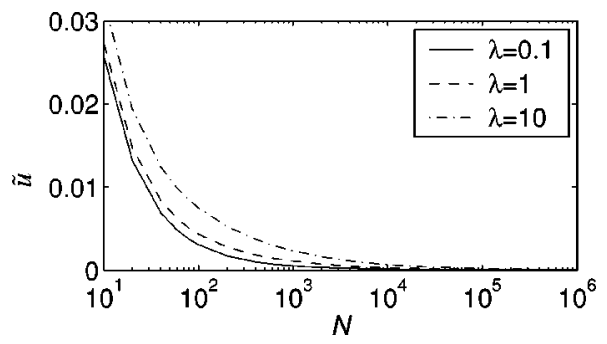

FIG. 1. The $N$ dependence of $\tilde{u}$ (in units of $\hbar \omega_{\perp}$ ) for a ${ }^{87} \mathrm{Rb}$ condensate in a cylindrically symmetric trap with $\omega_{x}=\omega_{y}=\omega_{\perp}$ $=(2 \pi) 100(\mathrm{~Hz})$ and $\omega_{z}=\lambda \omega_{\perp}$. We have used $a_{0}=101.8 a_{B}$ and $a_{2}=100.4 a_{B}$ ( $a_{B}$ is Bohr radius) [27].

time-dependent part in $\phi_{j}(\vec{r}, t)$ and $\theta_{j}(\vec{r}, t)$ from now on. The steady-state equations for phase functions now become

$$
\begin{aligned}
& {[\mathcal{L}-}\left.\mu+c_{0}\left(\rho+2 \rho_{+}\right)+c_{2}\left(3 \rho_{+}+\rho_{0}-\rho_{-}\right)\right] \xi_{+}+2 N\left(c_{+} \phi_{+}\right. \\
&\left.+c_{2} \phi_{-}\right) \phi_{0} \xi_{0}+N\left(2 c_{-} \phi_{+} \phi_{-}+c_{2} \phi_{0}^{2}\right) \xi_{-} \\
&= N \tilde{u} \phi_{+}, \\
& {\left[\mathcal{L}-\mu+c_{0}\left(\rho+2 \rho_{0}\right)+c_{2}\left(\rho_{+}+2 N \phi_{+} \phi_{-}+\rho_{-}\right)\right] \xi_{0} } \\
& \quad+2 N\left(c_{+} \phi_{+}+c_{2} \phi_{-}\right) \phi_{0} \xi_{+}+2 N\left(c_{+} \phi_{-}+c_{2} \phi_{+}\right) \phi_{0} \xi_{-} \\
&=N \tilde{u} \phi_{0}, \\
& {\left[\mathcal{L}-\mu+c_{0}\left(\rho+2 \rho_{-}\right)+c_{2}\left(3 \rho_{-}+\rho_{0}-\rho_{+}\right)\right] \xi_{-}+2 N\left(c_{+} \phi_{-}\right.} \\
&\left.\quad+c_{2} \phi_{+}\right) \phi_{0} \xi_{0}+N\left(2 c_{-} \phi_{+} \phi_{-}+c_{2} \phi_{0}^{2}\right) \xi_{+} \\
&=N \tilde{u} \phi_{-},
\end{aligned}
$$

where $\xi_{j}(\vec{r})=\theta_{j}(\vec{r}) e^{-i \alpha_{j}}$ are now real-valued functions. One can easily check that similar to the single mode approximation for $\phi_{j}(\vec{r}), \quad \xi_{j}(\vec{r})$ can also be expressed as $\xi_{j}(\vec{r})$ $=n_{j}^{1 / 2} \theta(\vec{r})$ with $\theta(\vec{r})$ governed by the following equation:

$$
\left[\mathcal{L}-\mu+3 c_{+} N \phi^{2}(\vec{r})\right] \theta(\vec{r})=N \tilde{u} \phi(\vec{r}),
$$

and $\tilde{u}$ determined through normalization constraint $\int d \vec{r} \phi(\vec{r}) \theta(\vec{r})=1 / 2$. Figure 1 shows the selected results for the $N$ dependence of $\tilde{u}$. Therefore, for ferromagnetic interactions, the phase functions can be generally expressed as

$$
\theta_{j}(\vec{r})=\sqrt{n_{j}} \theta(\vec{r}) e^{i \alpha_{j}}
$$

We note that Eq. (18) is, in fact, identical to the equation satisfied by the phase function of a scalar condensate [4]; a point easily understood for ferromagnetic interactions when all spins align along the same direction. If we take this direction as the quantization axis, then the spinor condensate is essentially a scalar condensate since its total magnetization $\mathcal{M}$ is conserved. All overlap integrals in Eq. (12) are presented in Appendix A. They are all real quantities with the choice of phase parameters.

We now attempt to express the zero mode dynamics in terms of its associated Hamiltonian. Since $P_{j}$ and $Q_{j}$ are not

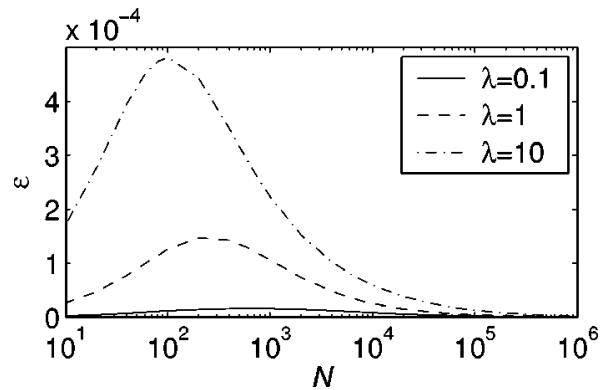

FIG. 2. The $N$ dependence of $\varepsilon$ (in units of $\hbar \omega_{\perp}$ ) for the same parameters as in Fig. 1.

canonically conjugated variables, we define $p_{j}^{\prime} \equiv P_{j}$ and $q_{j}^{\prime} \equiv Q_{j} / J_{j}$ such that they become canonical conjugates. The zero mode Hamiltonian that governs the dynamics of Eq. (12) is

$$
\begin{aligned}
\frac{H_{\text {zero }}=}{N}= & \left(\frac{\tilde{u}}{2 n_{+} \mathcal{O}_{\phi \theta}}-2 c_{+} \frac{1-n_{+}}{n_{+}}-2 c_{2} \frac{n_{-}}{n_{+}}\right) p_{+}^{\prime 2} \mathcal{O}_{\phi \theta} \\
& +\left(\frac{\tilde{u}}{2 n_{0} \mathcal{O}_{\phi \theta}}-2 c_{+} \frac{1-n_{0}}{n_{0}}-2 c_{2}\right) p_{0}^{\prime 2} \mathcal{O}_{\phi \theta} \\
& +\left(\frac{\tilde{u}}{2 n_{-} \mathcal{O}_{\phi \theta}}-2 c_{+} \frac{1-n_{-}}{n_{-}}-2 c_{2} \frac{n_{+}}{n_{-}}\right) p_{-}^{\prime 2} \mathcal{O}_{\phi \theta} \\
& +4\left[c_{+}+c_{2}\left(\frac{n_{-}}{n_{+}}\right)^{1 / 2}\right] p_{+}^{\prime} p_{0}^{\prime} \mathcal{O}_{\phi \theta} \\
& +4\left[c_{+}+c_{2}\left(\frac{n_{+}}{n_{-}}\right)^{1 / 2}\right] p_{-}^{\prime} p_{0}^{\prime} \mathcal{O}_{\phi \theta}+4 c_{0} p_{+}^{\prime} p_{-}^{\prime} \mathcal{O}_{\phi \theta} \\
& -\frac{c_{2} n_{0}^{2} \mathcal{O}_{\phi \phi}}{2 \hbar^{2}}\left(q_{+}^{\prime}-2 q_{0}^{\prime}+q_{-}^{\prime}\right)^{2} \\
= & \mathbf{p}^{\prime T} \mathcal{A} \mathbf{p}^{\prime}+\mathbf{q}^{\prime T} \mathcal{B q}^{\prime},
\end{aligned}
$$

where $\mathbf{p}^{\prime T}=\left(p_{+}^{\prime}, p_{0}^{\prime}, p_{-}^{\prime}\right), \mathbf{q}^{\prime T}=\left(q_{+}^{\prime}, q_{0}^{\prime}, q_{-}^{\prime}\right)$, and $\mathcal{A}$ and $\mathcal{B}$ are two Hermitian matrices whose elements are easily identified from the above equation. We also present, in Appendix $\mathrm{B}$, the zero mode Hamiltonian in the form of overlap integrals. To simplify notations, we have also defined $\mathcal{O}_{\phi \theta}$ $\equiv \int d \vec{r} \phi^{2}(\vec{r}) \theta^{2}(\vec{r})$ and $\mathcal{O}_{\phi \phi} \equiv \int d \vec{r} \phi^{4}(\vec{r})$. We find that matrix $\mathcal{B}$ is positive definite since $c_{2}<0$ for ferromagnetic spinor condensates. To show the positive definiteness of $\mathcal{A}$, we decompose $\tilde{u}$ as

$$
\tilde{u}=\varepsilon+4 c_{+} \mathcal{O}_{\phi \theta},
$$

where

$$
\varepsilon=\frac{2}{N} \int d \vec{r} \theta(\vec{r})\left[\mathcal{L}-\mu+c_{+} N \phi^{2}(\vec{r})\right] \theta(\vec{r})
$$

We note that $\varepsilon \equiv 0$ for noninteracting atoms $[\phi(\vec{r})=\theta(\vec{r})]$. However, it is nonzero in general and we can prove that $\varepsilon$ $>0$ since $\theta(\vec{r})$ is the solution of Eq. (18) (see Fig. 2 for the $N$ dependence of $\varepsilon$ ). By using Eq. (21), we then obtain 


$$
\mathbf{p}^{\prime T} \mathcal{A} \mathbf{p}^{\prime}=\frac{\varepsilon}{2}\left(\frac{p_{+}^{\prime 2}}{n_{+}}+\frac{p_{0}^{\prime 2}}{n_{0}}+\frac{p_{-}^{\prime 2}}{n_{-}}\right)+2 \mathcal{O}_{\phi \theta} \mathbf{p}^{\prime T} \mathcal{A}^{\prime} \mathbf{p}^{\prime},
$$

where

$$
\mathcal{A}^{\prime}=\left(\begin{array}{ccc}
c_{0}+\frac{4 c_{2} m}{(1+m)^{2}} & c_{0}+\frac{2 c_{2}}{1+m} & c_{0} \\
c_{0}+\frac{2 c_{2}}{1+m} & c_{0} & c_{0}+\frac{2 c_{2}}{1-m} \\
c_{0} & c_{0}+\frac{2 c_{2}}{1-m} & c_{0}-\frac{4 c_{2} m}{(1-m)^{2}}
\end{array}\right) .
$$

It is then simple to verify that $\mathcal{A}^{\prime}$ is semipositive definite, which guarantees that $\mathcal{A}$ is positive definite. Thus, the initial ground state, the mean-field symmetry breaking state with a coherent condensate amplitude $\sqrt{N} \phi_{j}(\vec{r})$, is stable. The associated quantum fluctuations of the atom numbers can be studied by the linearization approximation Eq. (3). We note that $\mathcal{B}$ is diagonalized by an orthogonal matrix

$$
\mathcal{U}=\left(\begin{array}{ccc}
1 / \sqrt{3} & 1 / \sqrt{3} & 1 / \sqrt{3} \\
1 / \sqrt{2} & 0 & -1 / \sqrt{2} \\
1 / \sqrt{6} & -2 / \sqrt{6} & 1 / \sqrt{6}
\end{array}\right),
$$

which motivates the introduction of new number and phase fluctuation operators according to $\mathbf{p}^{T}=\mathbf{p}^{\prime T} \mathcal{U}^{T}$ $=\left(p_{N}, p_{M}, p_{Y}\right)$ and $\mathbf{q}^{T}=\mathbf{q}^{\prime T} \mathcal{U}^{T}=\left(q_{N}, q_{M}, q_{Y}\right)$. Not surprisingly, this transformation recovers the underline symmetry of a spinor-1 condensate, as discovered recently in Ref. [28]. It turns out that $p_{N}, p_{M}$, and $p_{Y}$ represent, respectively, the fluctuations of the total number of atoms, the magnetization, and the hypercharge. $\mathcal{A}$ is not simultaneously diagonalized by $\mathcal{U}$, yet the zero mode Hamiltonian takes a much simpler form

$$
\begin{aligned}
\frac{H_{\text {zero }}}{N}= & a p_{N}^{2}+b p_{M}^{2}+c p_{Y}^{2}+\alpha p_{N} p_{Y}+\beta p_{M} p_{Y}+\gamma p_{N} p_{M} \\
& +\eta q_{Y}^{2}
\end{aligned}
$$

where all the coefficients are given in Appendix C. By replacing $p_{j}$ with $-i \hbar \partial / \partial q_{j}$, we obtain an eigenvalue equation for the distribution of the various fluctuations in the condensate ground state,

$$
\begin{aligned}
\frac{E}{N \hbar^{2}} \varphi= & -a \frac{\partial^{2} \varphi}{\partial q_{N}^{2}}-b \frac{\partial^{2} \varphi}{\partial q_{M}^{2}}-c \frac{\partial^{2} \varphi}{\partial q_{Y}^{2}}-\alpha \frac{\partial^{2} \varphi}{\partial q_{N} q_{Y}}-\beta \frac{\partial^{2} \varphi}{\partial q_{M} q_{Y}} \\
& -\gamma \frac{\partial^{2} \varphi}{\partial q_{N} q_{M}}+\frac{\eta}{\hbar^{2}} q_{Y}^{2} \varphi .
\end{aligned}
$$

We look for eigenfunctions of the form

$$
\chi\left(q_{Y}\right) e^{i\left(k_{N} q_{N}+k_{M} q_{M}\right)},
$$

which, upon substituting into Eq. (25), yields

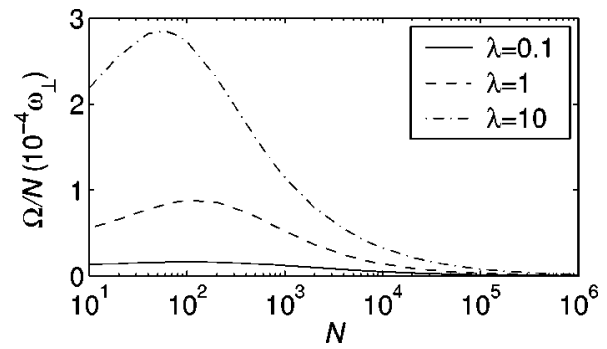

FIG. 3. The $N$ dependence of $\Omega / N$ for the same parameters as in Fig. 1.

$$
c \frac{d^{2} \chi}{d q_{Y}^{2}}+i \kappa \frac{d \chi}{d q_{Y}}+\left(E^{\prime}-\frac{\eta}{\hbar^{2}} q_{Y}^{2}\right) \chi=0
$$

with

$$
\begin{gathered}
\kappa=\alpha k_{N}+\beta k_{M} \\
E^{\prime}=\frac{E}{N \hbar^{2}}-\left(a k_{N}^{2}+b k_{M}^{2}+\gamma k_{N} k_{M}\right) .
\end{gathered}
$$

Eigenequation (27) is essentially of the harmonic type, which can be easily solved and the resulting eigenvalues of Eq. (25) are

$$
\frac{E_{n}}{N}=(2 n+1) \hbar(c \eta)^{1 / 2}+\Lambda\left(k_{N}, k_{M}\right),
$$

where $n=0,1,2, \ldots$ and

$$
\Lambda\left(k_{N}, k_{M}\right)=\hbar^{2}\left(a k_{N}^{2}+b k_{M}^{2}+\gamma k_{N} k_{M}\right)-\frac{\hbar^{2}}{4 c}\left(\alpha k_{N}+\beta k_{M}\right)^{2} .
$$

We note that $\Lambda\left(k_{N}, k_{M}\right)$ must be greater than or equal to zero due to the positive definiteness of matrix $\mathcal{A}$. Therefore, for the ground state of Eq. (25) we must have $k_{N}=k_{M}=0$, the ground-state $(n=0)$ energy and eigenfunction are, respectively,

$$
\begin{gathered}
E_{0}=N \hbar(c \eta)^{1 / 2} \equiv \frac{1}{2} \hbar \Omega, \\
\varphi_{0}\left(q_{Y}\right)=\pi^{-1 / 4}\left(\hbar \Delta_{Y 0}\right)^{-1 / 2} \exp \left(-\frac{q_{Y}^{2}}{2 \hbar^{2} \Delta_{Y 0}^{2}}\right),
\end{gathered}
$$

with

$$
\Omega=\frac{2 N}{\hbar}\left[-c_{2} \mathcal{O}_{\phi \phi}\left(\varepsilon-4 c_{2} \mathcal{O}_{\phi \theta}\right)\right]^{1 / 2}
$$

and

$$
\Delta_{Y 0}^{2}=\frac{1}{\hbar} \sqrt{\frac{c}{\eta}}=\frac{1}{3 n_{0}^{2}}\left(\frac{\varepsilon-4 c_{2} \mathcal{O}_{\phi \theta}}{-c_{2} \mathcal{O}_{\phi \phi}}\right)^{1 / 2} .
$$

We plotted the selected results for $\Omega / N$ and $\Delta_{Y 0}^{2}$ in Figs. 3 and 4 , respectively. 


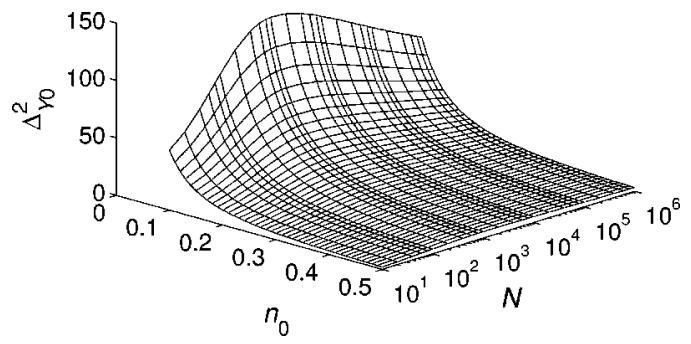

FIG. 4. Width $\Delta_{Y 0}^{2}$ of the ground-state distribution for $q_{Y}$, as a function of $n_{0}$ and $N$ for the same parameters as in Fig. 1 and $\lambda$ $=1$.

This ground state of $H_{\text {zero }}$ can be easily understood For a system starting with a fixed total number of atoms and magnetization, the dynamic conservation of the total number of atoms and total magnetization, as dictated by Hamiltonian (1), requires $\left\langle p_{N}^{2}\right\rangle=\left\langle p_{M}^{2}\right\rangle=0$, which leads to $\left\langle q_{N}^{2}\right\rangle=\left\langle q_{M}^{2}\right\rangle$ $=\infty$, i.e., the ground state of $H_{\text {zero }}$ has completely diffused phases for $q_{N}$ and $q_{M}$; the inherent ferromagnetic interaction, nevertheless, prepares a correlated ground state such that both $p_{Y}$ and $q_{Y}$ take a Gaussian form distribution with $\left\langle p_{Y}^{2}\right\rangle=1 /\left(2 \Delta_{Y 0}^{2}\right)$ and $\left\langle q_{Y}^{2}\right\rangle=\hbar^{2} \Delta_{Y 0}^{2} / 2$, which is, in fact, the minimal uncertainty coherent state that is consistent with the symmetry breaking mean-field assumptions of the ground state with three separate macroscopic wave functions for each of the spin components. However, it is experimentally difficult to produce a condensate having total fixed number and phase fluctuations as specified by such a state. We therefore turn to study the number and phase fluctuations of a general state. We start with the dynamic equations for $p_{j}$ and $q_{j}$

$$
\begin{gathered}
\dot{p}_{N}=0, \\
\dot{p}_{M}=0, \\
\dot{p}_{Y}=-2 N \eta q_{Y}, \\
\dot{q}_{N}=N\left(2 a p_{N}+\gamma p_{M}+\alpha p_{Y}\right), \\
\dot{q}_{M}=N\left(\gamma p_{N}+2 b p_{M}+\beta p_{Y}\right), \\
\dot{q}_{Y}=N\left(\alpha p_{N}+\beta p_{M}+2 c p_{Y}\right) .
\end{gathered}
$$

By noting that both $p_{N} \equiv p_{N}(0)$ and $p_{M} \equiv p_{M}(0)$ are constants of motion, we can express the solutions as

$$
\mathbf{x}(t)=\mathcal{T}(t) \mathbf{x}(0),
$$

where $\quad \mathbf{x}^{T}(t)=\left(p_{N}(t), p_{M}(t), p_{Y}(t), q_{N}(t), q_{M}(t), q_{Y}(t)\right)$, $\mathbf{x}^{T}(0)=\left(p_{N}(0), p_{M}(0), p_{Y}(0), q_{N}(0), q_{M}(0), q_{Y}(0)\right)$ is its initial value, and evolution matrix $\mathcal{T}(t)$ takes the following form:

$$
\mathcal{T}(t)=\left(\begin{array}{cccccc}
1 & 0 & 0 & 0 & 0 & 0 \\
0 & 1 & 0 & 0 & 0 & 0 \\
-\alpha^{\prime}(1-\cos \Omega t) & -\beta^{\prime}(1-\cos \Omega t) & \cos \Omega t & 0 & 0 & -\sin \Omega t / \hbar \Delta_{Y 0}^{2} \\
\zeta_{a} N t+\hbar \Delta_{Y 0}^{2} \alpha^{\prime 2} \sin \Omega t & \zeta_{c} N t+\hbar \Delta_{Y 0}^{2} \alpha^{\prime} \beta^{\prime} \sin \Omega t & \hbar \Delta_{Y 0}^{2} \alpha^{\prime} \sin \Omega t & 1 & 0 & -\alpha^{\prime}(1-\cos \Omega t) \\
\zeta_{c} N t+\hbar \Delta_{Y 0}^{2} \alpha^{\prime} \beta^{\prime} \sin \Omega t & \zeta_{b} N t+\hbar \Delta_{Y 0}^{2} \beta^{\prime 2} \sin \Omega t & \hbar \Delta_{Y 0}^{2} \beta^{\prime} \sin \Omega t & 0 & 1 & -\beta^{\prime}(1-\cos \Omega t) \\
\hbar \Delta_{Y 0}^{2} \alpha^{\prime} \sin \Omega t & \hbar \Delta_{Y 0}^{2} \beta^{\prime} \sin \Omega t & \hbar \Delta_{Y 0}^{2} \sin \Omega t & 0 & 0 & \cos \Omega t
\end{array}\right) .
$$

Coefficients $\alpha^{\prime}, \beta^{\prime}, \zeta_{a}, \zeta_{b}$, and $\zeta_{c}$ are given in Appendix D. The explicit solutions of the time-dependent number and phase fluctuations are given in Appendix E. We note that the general solution has a simple structure: in addition to oscillating terms of form $\cos \Omega t$ and $\sin \Omega t$, the phase fluctuations of $q_{N}$ and $q_{M}$ also contain terms proportional to $N t$. This indicates that our linearization approximation Eq. (3) is valid only for a finite duration. The time-dependent covariance can also be obtained to be

$$
\left\langle x_{i}(t) x_{j}(t)\right\rangle=\sum_{k l} \mathcal{T}_{i k}(t) \mathcal{T}_{j l}(t)\left\langle x_{k}(0) x_{l}(0)\right\rangle .
$$

Finally, let us consider the diffusion of the direction of macroscopic spin $\vec{f}(\vec{r}) \equiv \Sigma_{i j} \psi_{j}^{\dagger}(\vec{r}) \overrightarrow{\mathbf{F}}_{i j} \psi_{j}(\vec{r})$. As we have shown in Ref. [20], independent of the spatial coordinates, the spins of Bose condensed atoms are parallel for ferromag- netic interactions, i.e., it essentially acts as a macroscopic magnetic dipole pointing along the same direction,

$\vec{f}_{0}(\vec{r})=N \sum_{i j} \phi_{i}^{*}(\vec{r}) \overrightarrow{\mathbf{F}}_{i j} \phi_{j}(\vec{r})=N \phi^{2}(\vec{r})\left(\begin{array}{c}\sqrt{1-m^{2}} \cos \Theta \\ -\sqrt{1-m^{2}} \sin \Theta \\ m\end{array}\right)$,

where $\Theta=\alpha_{+}-\alpha_{0}=\alpha_{0}-\alpha_{-}$. Our initial choice of three separate macroscopic condensate wave functions (with given phases) for each of the spin components fixes the initial direction of the condensate spin direction. As the phase spreading dynamics attempts to restore the $\mathrm{U}(1)$ symmetries of each of the phases, our initial choice of phases will become irrelevant. Under linear approximation (3), the fluctuation becomes 


$$
\delta \vec{f}(t)=\int d \vec{r}\left[\vec{f}(\vec{r})-\vec{f}_{0}(\vec{r})\right],
$$

or, explicitly,

$$
\begin{aligned}
\delta f_{x}= & \frac{1}{\sqrt{2}} \int d \vec{r}\left(\phi_{0}^{*} \delta \psi_{+}+\phi_{+} \delta \psi_{0}^{\dagger}+\phi_{-}^{*} \delta \psi_{0}+\phi_{0} \delta \psi_{-}^{\dagger}\right. \\
& + \text { H.c. }), \\
\delta f_{y}= & \frac{i}{\sqrt{2}} \int d \vec{r}\left(\phi_{0}^{*} \delta \psi_{+}+\phi_{+} \delta \psi_{0}^{\dagger}+\phi_{-}^{*} \delta \psi_{0}+\phi_{0} \delta \psi_{-}^{\dagger}\right. \\
& - \text { H.c. }), \\
& \delta f_{z}=\int d \vec{r}\left(\phi_{+}^{*} \delta \psi_{+}-\phi_{-}^{*} \delta \psi_{-}+\text {H.c. }\right) .
\end{aligned}
$$

Although $\langle\delta \vec{f}(t)\rangle \equiv 0$, the dynamics of the zero mode causes the variance of $\delta \vec{f}(t)$ to be nonzero. It can be re-expressed in terms of the (time-dependent) number and phase fluctuation operators, under the RWA, as

$$
\begin{aligned}
& \delta f_{x}(t)= \frac{\cos \Theta}{\sqrt{1-m^{2}}}\left[\sqrt{3} p_{N}(t)-\sqrt{2} m p_{M}(t)\right] \\
&+\frac{\sqrt{1-m^{2}} \sin \Theta}{2 \hbar}\left[\sqrt{2} q_{M}(t)+\sqrt{6} m q_{Y}(t)\right], \\
& \delta f_{y}(t)= \frac{-\sin \Theta}{\sqrt{1-m^{2}}}\left[\sqrt{3} p_{N}(t)-\sqrt{2} m p_{M}(t)\right] \\
&+\frac{\sqrt{1-m^{2}} \cos \Theta}{2 \hbar}\left[\sqrt{2} q_{M}(t)+\sqrt{6} m q_{Y}(t)\right], \\
& \delta f_{z}(t)=\sqrt{2} p_{M}(t),
\end{aligned}
$$

where the time-dependent terms are

$$
\begin{aligned}
\sqrt{2} q_{M}(t)+\sqrt{6} m q_{Y}(t)= & \sqrt{2} q_{M}(0)+\sqrt{6} m q_{Y}(0)+\frac{4 \varepsilon N t}{1-m^{2}} \\
& \times\left[-\sqrt{3} m p_{N}(0)+\sqrt{2} p_{M}(0)\right] .
\end{aligned}
$$

We see that $\delta f_{z}$ is a constant of motion due to the conservation of total magnetization. On the other hand, both $\delta f_{x}$ and $\delta f_{y}$ diffuse with time. To calculate the variance of $\delta \vec{f}(t)$, we simply take $\Theta=0$, which yields

$$
\begin{aligned}
\delta f_{x}(t) & =\frac{1}{\sqrt{1-m^{2}}}\left[\sqrt{3} p_{N}(0)-\sqrt{2} m p_{M}(0)\right], \\
\delta f_{y}(t) & =\frac{\sqrt{1-m^{2}}}{2 \hbar}\left[\sqrt{2} q_{M}(t)+\sqrt{6} m q_{Y}(t)\right],
\end{aligned}
$$

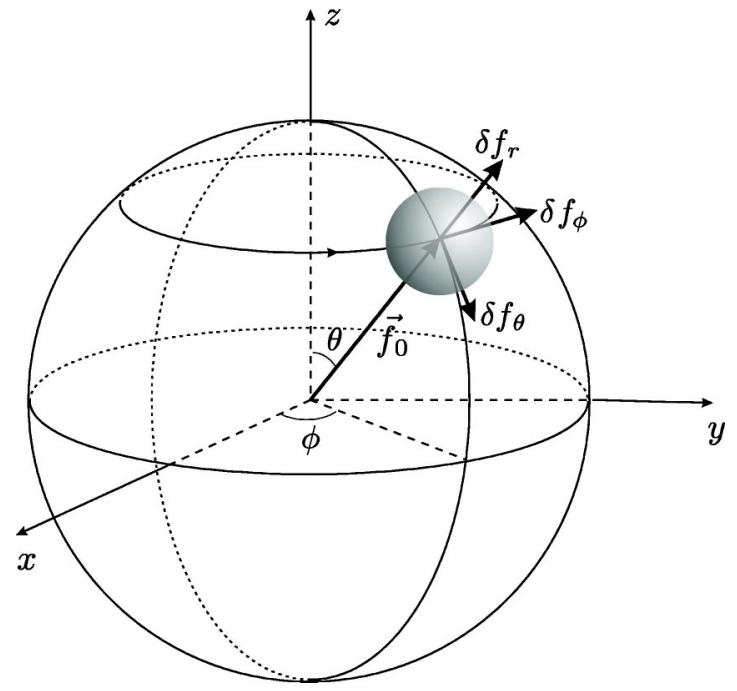

FIG. 5. Illustration of the macroscopic condensate spin and its fluctuation in spherical coordinates.

$$
\delta f_{z}(t)=\sqrt{2} p_{M}(0),
$$

i.e., with the direction of the initial macroscopic spin constrained in the $x z$ plane. The results of the variances for condensate spin direction fluctuations are listed in Appendix F.

We can also project the fluctuation of the spin [Eq. (38)] onto spherical coordinates $(\hat{r}, \hat{\theta}, \hat{\phi})$, as illustrated in Fig. 5, and noting that

$$
\begin{gathered}
\hat{r}=\hat{x} \sin \theta \cos \phi+\hat{y} \sin \theta \sin \phi+\hat{z} \cos \theta, \\
\hat{\theta}=\hat{x} \cos \theta \cos \phi+\hat{y} \cos \theta \sin \phi-\hat{z} \sin \theta, \\
\hat{\phi}=-\hat{x} \sin \phi+\hat{y} \cos \phi,
\end{gathered}
$$

and

$$
\begin{gathered}
\sin \theta=\sqrt{1-m^{2}}, \quad \cos \theta=m, \\
\sin \phi=-\sin \Theta, \quad \cos \phi=\cos \Theta,
\end{gathered}
$$

we find

$$
\begin{gathered}
\delta f_{r}(t)=\sqrt{3} p_{N}(0), \\
\delta f_{\theta}(t)=\frac{1}{\sqrt{1-m^{2}}}\left[\sqrt{3} m p_{N}(0)-\sqrt{2} p_{M}(0)\right], \\
\delta f_{\phi}(t)=\frac{\sqrt{1-m^{2}}}{2 \hbar}\left[\sqrt{2} q_{M}(t)+\sqrt{6} m q_{Y}(t)\right] .
\end{gathered}
$$

We immediately see that both $\delta f_{r}(t) \equiv \delta f_{r}(0)$ and $\delta f_{\theta}(t)$ $\equiv \delta f_{\theta}(0)$ are fixed due to the conservations of $N$ and $\mathcal{M}$. Furthermore, with the use of Eq. (39), we find that $\delta f_{\phi}(t)$ can be rewritten as

$$
\delta f_{\phi}(t)=\delta f_{\phi}(0)-\frac{2 \varepsilon N t}{\hbar} \delta f_{\theta}(0),
$$


where

$$
\delta f_{\phi}(0)=\frac{\sqrt{1-m^{2}}}{2 \hbar}\left[\sqrt{2} q_{M}(0)+\sqrt{6} m q_{Y}(0)\right] .
$$

We then see that $\delta f_{\phi}(t)$ grows linearly with $t$ and the diffusion rate is given by

$$
R_{d}=-\frac{2 \varepsilon N}{\hbar} \delta f_{\theta}(0)
$$

which is proportional to $\delta f_{\theta}(0)$. A result easily understandable in terms of the single axis twisting of isospin $T_{z}^{2}$ (of the Hamiltonian) of a spinor-1 condensate [28].

Given any initial condensate state, its variances in atom numbers, for each individual spin component and their correlations, and related phase fluctuations completely determine the subsequent fluctuations of $\delta f_{\phi}(t) . P_{j}(0)$ is, in fact, nothing but the atom number fluctuation of state $|j\rangle$. In general, we we note that

$$
\begin{aligned}
\int d \vec{r} \psi_{j}^{\dagger}(\vec{r}) \psi_{j}(\vec{r}) & =N+\sqrt{N} P_{j}+\int d \vec{r} \delta \psi_{j}^{\dagger}(\vec{r}) \delta \psi_{j}(\vec{r}) \\
& =N+\sqrt{N} P_{j}+\frac{s n_{j}}{2 J_{j}^{2}} P_{j}^{2}+\frac{n_{j}}{2 \hbar^{2} J_{j}^{2}} Q_{j}^{2}-\frac{1}{2}
\end{aligned}
$$

where $s=\int d \vec{r} \theta^{2}(\vec{r})$ and we have used RWA in deriving the last line. One can easily find

$$
\begin{aligned}
\delta N_{j}^{2} & =\left\langle N_{j}^{2}\right\rangle-\left\langle N_{j}\right\rangle^{2} \\
& =\left\langle\left[\int d \vec{r} \psi_{j}^{\dagger}(\vec{r}) \psi_{j}(\vec{r})\right]^{2}\right\rangle-\left\langle\int d \vec{r} \psi_{j}^{\dagger}(\vec{r}) \psi_{j}(\vec{r})\right\rangle^{2} \\
& \approx N\left\langle P_{j}^{2}\right\rangle \\
& =N\left\langle p_{j}^{\prime 2}\right\rangle,
\end{aligned}
$$

to lowest order.

If we use $\sigma_{N}^{2}, \sigma_{M}^{2}$, and $\sigma_{Y}^{2}$ to denote the variances of the total atom number $(N)$, the magnetization $(\mathcal{M})$, and the hypercharge $(Y)$, we find that

$$
\begin{gathered}
\left\langle\left[\delta f_{r}(t)\right]^{2}\right\rangle=3 \sigma_{N}^{2}, \\
\left\langle\left[\delta f_{\theta}(t)\right]^{2}\right\rangle=\frac{1}{1-m^{2}}\left(3 m^{2} \sigma_{N}^{2}+2 \sigma_{M}^{2}\right) .
\end{gathered}
$$

To calculate the variance of $\delta f_{\phi}(t)$, we assume a completely uncorrelated (between fluctuations of $N, \mathcal{M}$, and $Y$ ) initial distribution in the form of a Gaussian wave packet

$$
\begin{aligned}
\varphi\left(p_{N}, p_{M}, p_{Y}\right)= & (2 \pi)^{-3 / 4}\left(\sigma_{N} \sigma_{M} \sigma_{Y}\right)^{-1 / 2} \\
& \times \exp \left[-\frac{1}{4}\left(\frac{p_{N}^{2}}{\sigma_{N}^{2}}+\frac{p_{M}^{2}}{\sigma_{M}^{2}}+\frac{p_{Y}^{2}}{\sigma_{Y}^{2}}\right)\right] .
\end{aligned}
$$

Since $q_{j}=i \hbar \partial / \partial p_{j}$, we find, for such a distribution,

$$
\begin{gathered}
\left\langle q_{i}(0) q_{j}(0)\right\rangle=\frac{\hbar^{2}}{4 \sigma_{i}^{2}} \delta_{i j}, \\
\left\langle p_{i}(0) p_{j}(0)\right\rangle=\sigma_{i}^{2} \delta_{i j}, \\
\left\langle q_{i}(0) p_{i}(0)+p_{i}(0) q_{i}(0)\right\rangle=0, \\
\left\langle q_{i}(0) p_{j}(0)\right\rangle=0 \text { for } i \neq j,
\end{gathered}
$$

and $i, j=N, M, Y$. Therefore,

$$
\left\langle\left[\delta f_{\phi}(t)\right]^{2}\right\rangle=\left\langle\left[\delta f_{\phi}(0)\right]^{2}\right\rangle+\frac{4 \varepsilon^{2} N^{2} t^{2}}{\hbar^{2}}\left\langle\left[\delta f_{\theta}(0)\right]^{2}\right\rangle,
$$

with

$$
\left\langle\left[\delta f_{\phi}(0)\right]^{2}\right\rangle=\frac{1-m^{2}}{8}\left(\frac{1}{\sigma_{M}^{2}}+3 m^{2} \frac{1}{\sigma_{Y}^{2}}\right) .
$$

If, on the other hand, we take a distribution similar to Eq. (48) but with no correlations between the populations in the spin components $j=+, 0,-$,

$$
\begin{aligned}
\varphi\left(p_{+}^{\prime}, p_{0}^{\prime}, p_{-}^{\prime}\right)= & (2 \pi)^{-3 / 4}\left(\sigma_{+} \sigma_{0} \sigma_{-}\right)^{-1 / 2} \\
& \times \exp \left[-\frac{1}{4}\left(\frac{p_{+}^{\prime 2} 2}{\sigma_{+}^{2}}+\frac{p_{0}^{\prime 2}}{\sigma_{0}^{2}}+\frac{p_{-}^{\prime 2}}{\sigma_{-}^{2}}\right)\right],
\end{aligned}
$$

with $\sigma_{j}^{2}$ denoting the corresponding atom number variance, we find

$$
\begin{gathered}
\left\langle\left[\delta f_{r}(t)\right]^{2}\right\rangle=\sigma_{+}^{2}+\sigma_{0}^{2}+\sigma_{-}^{2}, \\
\left\langle\left[\delta f_{\theta}(t)\right]^{2}\right\rangle=\frac{1}{1-m^{2}}\left[(1-m)^{2} \sigma_{+}^{2}+m^{2} \sigma_{0}^{2}+(1+m)^{2} \sigma_{-}^{2}\right],
\end{gathered}
$$

and Eq. (50) still holds but with

$$
\left\langle\left[\delta f_{\phi}(0)\right]^{2}\right\rangle=\frac{1-m^{2}}{16}\left[\frac{(1+m)^{2}}{\sigma_{+}^{2}}+4 m^{2} \frac{1}{\sigma_{0}^{2}}+\frac{(1-m)^{2}}{\sigma_{-}^{2}}\right] .
$$

In the Thomas-Fermi (TF) regime, we obtain most of the above results analytically. First we note that the ground wave function is

$$
\phi^{2}(\vec{r})=\left\{\begin{array}{cc}
(c+N)^{-1}\left[\mu-V_{\mathrm{ext}}(\vec{r})\right] & \text { if } \mu>V_{\mathrm{ext}}(\vec{r}), \\
0 & \text { otherwise }
\end{array}\right.
$$

where $c_{+}=4 \pi \hbar^{2} a_{2} / M$ and chemical potential

$$
\mu=\frac{\hbar \omega_{\mathrm{ho}}}{2}\left(\frac{15 N a_{2}}{a_{\mathrm{ho}}}\right)^{2 / 5}
$$


with $\omega_{\text {ho }}=\left(\omega_{x} \omega_{y} \omega_{z}\right)^{1 / 3}$ and $a_{\mathrm{ho}}=\left(\hbar / M \omega_{\mathrm{ho}}\right)^{1 / 2}$. Similarly, ignoring the kinetic-energy term in Eq. (18), we obtain

$$
\theta(\vec{r})=\left\{\begin{array}{cc}
{\left[2 V_{0} \phi(\vec{r})\right]^{-1}} & \text { if } \mu>V_{\text {ext }}(\vec{r}), \\
0 & \text { otherwise }
\end{array}\right.
$$

and

$$
\tilde{u}=\frac{c_{+}}{V_{0}}
$$

with

$$
V_{0}=\int_{\mu-V_{\mathrm{ext}}(\vec{r})>0} d \vec{r}=\frac{4 \pi a_{\mathrm{ho}}^{3}}{3}\left(\frac{15 a_{2}}{a_{\mathrm{ho}}}\right)^{3 / 5} N^{3 / 5} .
$$

All overlap integrals can be calculated analytically as

$$
\begin{gathered}
\mathcal{O}_{\phi \theta}=\left(4 V_{0}\right)^{-1}=\frac{3}{16 \pi a_{\mathrm{ho}}^{3}}\left(\frac{a_{\mathrm{ho}}}{15 a_{2}}\right)^{3 / 5} N^{-3 / 5}, \\
\mathcal{O}_{\phi \phi}=\frac{32 \pi \mu^{7 / 2}}{105 c_{+}^{2} N^{2} \omega_{\mathrm{ho}}^{3}}\left(\frac{2}{M}\right)^{3 / 2}=\frac{15^{2 / 5}}{14 \pi a_{\mathrm{ho}}^{3}}\left(\frac{a_{\mathrm{ho}}}{a_{2}}\right)^{3 / 5} N^{-3 / 5} .
\end{gathered}
$$

Parameter $\varepsilon$ relates essentially to kinetic-energy operator $E_{k}^{(\theta)}=-\left(\hbar^{2} / 2 M\right) \int d \vec{r} \theta \nabla^{2} \theta$, evaluated with respect to phase function $\theta(\vec{r})$. In the TF approximation we find

$$
N \varepsilon=2 E_{k}^{(\theta)} .
$$

Using Eqs. (15) and (18) we find

$$
\left(\mathcal{L}-\mu+3 c_{+} N \phi^{2}\right) \theta=\frac{N \tilde{u}}{\mu}\left(\mathcal{L}+c_{+} N \phi^{2}\right) \phi,
$$

which simplifies to

$$
\left(-\frac{\hbar^{2} \nabla^{2}}{2 M}+2 c_{+} N \phi^{2}\right) \theta=\frac{N \tilde{u}}{\mu}\left(-\frac{\hbar^{2} \nabla^{2}}{2 M}+\mu\right) \phi,
$$

in the TF limit. Multiplying Eq. (60) from left by $\theta$ and $\phi$, respectively, and integrating over $\vec{r}$, we obtain

$$
\begin{gathered}
E_{k}^{(\theta)}+2 c_{+} N \mathcal{O}_{\phi \theta}=\frac{N \tilde{u}}{\mu}\left(E_{k}^{(\phi \theta)}+\frac{\mu}{2}\right), \\
E_{k}^{(\phi \theta)}+2 c_{+} N \int d \vec{r} \phi^{3} \theta=\frac{N \tilde{u}}{\mu}\left(E_{k}^{(\phi)}+\mu\right),
\end{gathered}
$$

where $E_{k}^{(\phi)}=-\left(\hbar^{2} / 2 M\right) \int d \vec{r} \phi \nabla^{2} \phi$ is the kinetic energy per atom in the condensate and $E_{k}^{(\phi \theta)}=-\left(\hbar^{2} / 2 M\right) \int d \vec{r} \phi \nabla^{2} \theta$ $=-\left(\hbar^{2} / 2 M\right) \int d \vec{r} \theta \nabla^{2} \phi$. Eliminating $E_{k}^{(\phi \theta)}$ from above two equations, we obtain

$$
\begin{aligned}
E_{k}^{(\theta)}= & \left(\frac{N \tilde{u}}{\mu}\right)^{2} E_{k}^{(\phi)}+\frac{N \tilde{u}}{2}-2 c_{+} N \mathcal{O}_{\phi \theta} \\
& +\frac{N \tilde{u}}{\mu}\left(N \tilde{u}-2 c_{+} N \int d \vec{r} \phi^{3} \theta\right) \\
= & \left(\frac{N \tilde{u}}{\mu}\right)^{2} E_{k}^{(\phi)} \\
= & \frac{4}{25} E_{k}^{(\phi)} .
\end{aligned}
$$

For a spherical trap, adopting the result of Ref. [29], we immediately find

$$
\varepsilon=\frac{4 \hbar^{2}}{5 M N R^{2}} \ln \left(\frac{R}{1.3 a_{\mathrm{ho}}}\right)
$$

with

$$
R=\left(15 a_{2} a_{\mathrm{ho}}^{4}\right)^{1 / 5} N^{1 / 5},
$$

which seems to indicate that $N \varepsilon \propto N^{-2 / 5}$ asymptotically goes to zero at large $N$. By all measures, we find $\varepsilon$ to be a very small quantity as compared to $c_{2} \mathcal{O}_{\phi \theta}$. Taking $\varepsilon$ as zero in the TF limit, we obtain

$$
\begin{gathered}
n_{0}^{2} \Delta_{Y 0}^{2}=\frac{1}{3} \sqrt{\frac{7}{10}}, \\
\hbar \Omega=-\frac{\sqrt{3 / 14}}{15^{1 / 10}}\left(\frac{c_{2}}{\pi a_{\mathrm{ho}}^{3}}\right)\left(\frac{a_{\mathrm{ho}}}{a_{2}}\right)^{3 / 5} N^{2 / 5} .
\end{gathered}
$$

To shed light on the above scaling results based on the TF approximation, we have performed extensive numerical calculations. As an example, we consider a spinor condensate of ${ }^{87} \mathrm{Rb}$ atoms in a spherically symmetric trap with a harmonic frequency $\omega=(2 \pi) 100(\mathrm{~Hz})$ and consider the regime of $N$ $\sim 10^{7}-10^{15}$. It turns out that our results indeed confirm the scaling relationship

$$
\mathcal{O}_{\phi \phi}, \mathcal{O}_{\phi \theta}, \tilde{u}, \Omega / N \propto N^{-3 / 5}
$$

and

$$
\begin{gathered}
\frac{N \tilde{u}}{\mu} \rightarrow \frac{2}{5}, \\
n_{0}^{2} \Delta_{Y 0}^{2} \rightarrow \frac{1}{3} \sqrt{\frac{7}{10}},
\end{gathered}
$$

in the large- $N$ limit. We also find that

$$
E_{k}^{(\phi)} \propto N^{-0.3549}
$$

in rough agreement with the result of Ref. [29]; On the other hand, we find 


$$
N \varepsilon \propto N^{0.1348}, \quad E_{k}^{(\theta)} \propto N^{0.1314},
$$

and

$$
N \varepsilon \approx 4 E_{k}^{(\theta)}
$$

which do not agree with the results above, as obtained with the TF approximation. A careful analysis, as confirmed by our numerical results, reveals that although $\int d \vec{r}\left(V_{\text {ext }}-\mu\right.$ $\left.+c_{+} N \phi^{2}\right) \phi^{2}$ is small, $\int d \vec{r}\left(V_{\text {ext }}-\mu+c_{+} N \phi^{2}\right) \theta^{2}$ is not, and, approximately, we find

$$
E_{k}^{(\theta)} \approx \frac{1}{2} \int d \vec{r}\left(V_{\mathrm{ext}}-\mu+c_{+} N \phi^{2}\right) \theta^{2} .
$$

This leads to the opposite $N$ dependence for $E_{k}^{(\theta)}$ and $E_{k}^{(\phi)}$; while $E_{k}^{(\theta)}$ increases with increasing values of $N, E_{k}^{(\phi)}$ decreases. Thus, identity Eq. (62), as obtained in the TF limit, is invalid.

\section{A NONZERO MAGNETIC FIELD}

Inside a nonzero homogeneous magnetic field $(\vec{B})$, the atomic quantization axis becomes fixed, along the direction of $\vec{B}$, or more conveniently denoted as the $z$ axis. Mathematically, it turns out that this is equivalent to the requirement of the conservation of magnetization $\mathcal{M}=N_{+}-N_{-}$. This reduces the $\mathrm{SO}(3)$ rotational symmetry to its subgroup $\mathrm{SO}(2)$, which is still a continuous symmetry. Thus, the quantum dynamics of the phase remains formally the same as discussed before.

In the linear Zeeman regime, the original Hamiltonian (1) is augmented to

$$
H^{\prime}=H-\hbar \omega_{L} \mathbf{F}_{z}
$$

The Larmor precessing frequency is $\omega_{L}=B \mu_{B} / \hbar$ with $\mu_{B}$ being the magnetic dipole moment for state $\left|F=1, M_{F}=1\right\rangle$. In this case, the only change is the phases of the spatial wave functions and the phase functions, if of the following timedependent form:

$$
\alpha_{j}=\alpha_{j 0}+j \omega_{L} t
$$

for $j=+, 0$, and,$- \alpha_{+0}-\alpha_{00}=\alpha_{00}-\alpha_{-0}=0$ still holds true for a ferromagnetic spinor-1 condensate. A direct consequence of the magnetic field is thus a time-dependent relative phase

$$
\Theta(t)=\alpha_{+0}-\alpha_{00}+\omega_{L} t=\alpha_{00}-\alpha_{-0}+\omega_{L} t .
$$

When applied to the macroscopic condensate spin, we find that the spin gains a precession around the $z$ axis.

Now the picture of the dynamics of the macroscopic condensate spin is clear: besides the precession induced by the external $B$ field, it also diffuses to restore the $\mathrm{U}(1)$ and $\mathrm{SO}(2)$ symmetries of the Hamiltonian.

The effect of quadratic Zeeman shift can also be simply addressed. Apart from an overall shift, it introduces a (positive) $B$-field dependent level shift $\propto \hbar \delta_{B} F_{z}^{2}$ to states $| \pm\rangle$ with respect to state $|0\rangle$. This differential shift causes the precessing of the condensate spin to be twisted, i.e., the precessing of the $|+\rangle(|-\rangle)$ component being slower (faster) by $\delta_{B}$.

\section{THE CASE OF ANTIFERROMAGNETIC INTERACTIONS}

Our discussion of the phase diffusion in the preceding section is based on the proof that the single spatial mode approximation is exact for ferromagnetic interactions. One might, therefore, conclude that a similar study for antiferromagnetic interactions would be difficult as, unlike for ferromagnetic interactions, it is difficult to predict the groundstate condensate structure, although it is clear that the SMA becomes invalid, in general [20]. Under the SMA, other studies $[30,31]$ have indicated that the ground state could become fragmented when the external magnetic field is negligibly small. Adopting the mean-field ground state, however, the quantum phase problem for antiferromagnetic interactions is much simpler.

First we note that when $\mathcal{M} \neq 0$, the population in the 0 th component is zero in the ground state $[20,24]$. Therefore, the zero mode Hamiltonian (20) reduces to

$$
\begin{aligned}
\frac{H_{\mathrm{zero}}}{N}= & -\frac{P_{+}^{2}}{J_{+}^{2}}\left(-\frac{J_{+}}{2} \tilde{u}+2 c_{-} I_{-+}\right)-\frac{P_{-}^{2}}{J_{-}^{2}}\left(-\frac{J_{-}}{2} \tilde{u}\right. \\
& \left.+2 c_{-} I_{-+}\right)+\frac{4 c_{-} P_{+} P_{-}}{J_{+} J_{-}} I_{-+},
\end{aligned}
$$

which is exactly the same zero mode Hamiltonian for a binary condensate with no coupling between its two components. This has already been studied [7]. We note that the results obtained in Sec. II remain valid.

Now, let's consider the $\mathcal{M}=0$ case. As was pointed out in Ref. [20], the single mode approximation again applies in this case, and we can assume

$$
\phi_{j}(\vec{r})=\sqrt{n_{j}} \phi(\vec{r}) e^{i \alpha_{j}},
$$

where $n_{+}=n_{-}=\left(1-n_{0}\right) / 2$ and total energy of the system is independent of the value of $n_{0}$. A similar relation exists among the phases of the three components: $\alpha_{+}+\alpha_{-}-2 \alpha_{0}$ $=\pi$. Spatial profile $\phi(\vec{r})$ satisfies the following equation:

$$
\left[\mathcal{L}+c_{0} N \phi^{2}(\vec{r})\right] \phi(\vec{r})=\mu \phi(\vec{r}),
$$

with a coefficient $c_{0}$ for the nonlinear term. The phase function then becomes

$$
\theta_{j}(\vec{r})=\sqrt{n_{j}} \theta(\vec{r}) e^{i \alpha_{j}}
$$

where $\theta(\vec{r})$ is the solution of the following equation:

$$
\left[\mathcal{L}-\mu+3 c_{0} N \phi^{2}(\vec{r})\right] \theta(\vec{r})=N \tilde{u} \theta(\vec{r}) .
$$

Following the results of Sec. III, we define

$$
\varepsilon=\frac{2}{N} \int d \vec{r} \theta(\vec{r})\left[\mathcal{L}-\mu+c_{0} N \phi^{2}(\vec{r})\right] \theta(\vec{r}),
$$

and the zero mode Hamiltonian becomes 


$$
\begin{aligned}
\frac{H_{\text {zero }}}{N}= & {\left[\frac{\varepsilon}{\left(1-n_{0}\right) \mathcal{O}_{\phi \theta}}+2 c_{0}+\frac{2 c_{2}}{1-n_{0}}\right] p_{+}^{\prime 2} \mathcal{O}_{\phi \theta}+\left(\frac{\varepsilon}{2 n_{0} \mathcal{O}_{\phi \theta}}\right.} \\
& \left.+2 c_{0}\right) p_{0}^{\prime 2} \mathcal{O}_{\phi \theta}+\left[\frac{\varepsilon}{\left(1-n_{0}\right) \mathcal{O}_{\phi \theta}}+2 c_{0}\right. \\
& \left.+\frac{2 c_{2}}{1-n_{0}}\right] p_{-}^{\prime 2} \mathcal{O}_{\phi \theta}+4 c_{0} p_{+}^{\prime} p_{0}^{\prime} \mathcal{O}_{\phi \theta}+4 c_{0} p_{-}^{\prime} p_{0}^{\prime} \mathcal{O}_{\phi \theta} \\
& +4\left(c_{0}+c_{2} \frac{2 n_{0}-1}{1-n_{0}}\right) p_{+}^{\prime} p_{-}^{\prime} \mathcal{O}_{\phi \theta} \\
& +\frac{c_{2} n_{0}\left(1-n_{0}\right) \mathcal{O}_{\phi \phi}}{2 \hbar^{2}}\left(q_{+}^{\prime}-2 q_{0}^{\prime}+q_{-}^{\prime}\right)^{2} \\
= & \mathbf{p}^{\prime T} \mathcal{A} \mathbf{p}^{\prime}+\mathbf{q}^{\prime} \mathcal{B q}^{\prime} .
\end{aligned}
$$

The positive definiteness of matrices $\mathcal{A}$ and $\mathcal{B}$ can again be verified. By applying the same transformation (23), we obtain

$$
\frac{H_{\text {zero }}}{N}=a p_{N}^{2}+b p_{M}^{2}+c p_{Y}^{2}+\alpha p_{N} p_{Y}+\eta q_{Y}^{2},
$$

where all coefficients are now given in Appendix G. We see that fluctuations in $N$ are completely decoupled from that of
$M$ and $Y$ in this case, a result that also happens for ferromagnetic interactions when $\mathcal{M}=0$. The ground state of Eq. (76) is similar to Eq. (20), except that now

$$
\begin{gathered}
\Omega=2 N \sqrt{c \eta}=\frac{2 N}{\hbar}\left[c_{2} \mathcal{O}_{\phi \phi}\left(\varepsilon+4 c_{2} n_{0}^{2} \mathcal{O}_{\phi \theta}\right)\right]^{1 / 2}, \\
\Delta_{Y 0}^{2}=\frac{1}{\hbar} \sqrt{\frac{c}{\eta}}=\frac{1}{3}\left[\frac{\varepsilon+4 c_{2} n_{0}^{2} \mathcal{O}_{\phi \theta}}{c_{2} n_{0}^{2}\left(1-n_{0}\right)^{2} \mathcal{O}_{\phi \phi}}\right]^{1 / 2} .
\end{gathered}
$$

The dynamic equations for $p_{i}$ and $q_{i}$ then become

$$
\begin{gathered}
\dot{p}_{N}=0, \\
\dot{p}_{M}=0, \\
\dot{p}_{Y}=-2 N \eta q_{Y}, \\
\dot{q}_{N}=N\left(2 a p_{N}+\alpha p_{Y}\right), \\
\dot{q}_{M}=2 N b p_{M}, \\
\dot{q}_{Y}=N\left(\alpha p_{N}+2 c p_{Y}\right) .
\end{gathered}
$$

One can easily find the evolution matrix to be

$\left.\begin{array}{cccc}0 & 0 & 0 & 0 \\ 0 & 0 & 0 & 0 \\ \cos \Omega t & 0 & 0 & -\sin \Omega t / \hbar \Delta_{Y 0}^{2} \\ \hbar \Delta_{Y 0}^{2} \alpha^{\prime} \sin \Omega t & 1 & 0 & -\alpha^{\prime}(1-\cos \Omega t) \\ 0 & 0 & 1 & 0 \\ \hbar \Delta_{Y 0}^{2} \sin \Omega t & 0 & 0 & \cos \Omega t\end{array}\right)$,

where

$$
\begin{gathered}
\alpha^{\prime}=\frac{\alpha}{2 c}=\frac{\left(3 n_{0}-1\right) \varepsilon+8 c_{2} n_{0}^{2} \mathcal{O}_{\phi \theta}}{\sqrt{2}\left(\varepsilon+4 c_{2} n_{0}^{2} \mathcal{O}_{\phi \theta}\right)}, \\
\zeta_{a}=\frac{4 a c-\alpha^{2}}{2 c}=12 c_{0} \mathcal{O}_{\phi \theta}+\frac{3 \varepsilon\left(\varepsilon+4 c_{2} n_{0} \mathcal{O}_{\phi \theta}\right)}{\varepsilon+4 c_{2} n_{0}^{2} \mathcal{O}_{\phi \theta}} .
\end{gathered}
$$

As for $\delta \vec{f}(t)$, we find

$$
\begin{gathered}
\delta f_{x}=\cos \Theta \sqrt{\frac{2 n_{0}}{1-n_{0}}} p_{M}+\sin \Theta \frac{\sqrt{6 n_{0}\left(1-n_{0}\right)}}{\hbar} q_{Y}, \\
\delta f_{y}=-\sin \Theta \sqrt{\frac{2 n_{0}}{1-n_{0}}} p_{M}+\cos \Theta \frac{\sqrt{6 n_{0}\left(1-n_{0}\right)}}{\hbar} q_{Y}, \\
\delta f_{z}=\sqrt{2} p_{M} .
\end{gathered}
$$

\section{CONCLUSIONS}

In conclusion, we have investigated, in detail, quantum phase diffusions of a spinor- 1 condensate. When the elastic atomic interaction is of ferromagnetic type, the structure of the ground state is greatly simplified: the spatial mode functions of the different spin components are exactly the same and accordingly, we also proved that the phase functions are exactly the same. This simplification allows us to construct the zero mode Hamiltonian which describes the number and phase fluctuations of a condensate while maintaining the conservations of the total number of atoms and the total magnetization. We have provided analytical results for the number and phase fluctuations due to both the quantum phase diffusion dynamics and the initial distribution of atom number and phase fluctuations. The structure of these fluctuations is rather simple: along with the oscillations due to $\sin \Omega t$ and $\cos \Omega t$ terms, quantum phase diffusion terms proportional to $N t$ also exist. This suggests that the mean-field 
approach to spinor-1 condensates is only valid for a finite duration. Based on these results, we have studied the diffusion of the direction of a macroscopic condensate spin. Our investigation sheds important light on the studies of quantum correlations, beyond mean-field theory, among Bose condensed atoms.

\section{ACKNOWLEDGMENTS}

We thank Yueheng Lan and Dr. X. X. Yi for helpful discussions. This work was supported by NSF and by a grant from the National Security Agency (NSA), Advanced Research and Development Activity (ARDA), and the Defense Advanced Research Projects Agency (DARPA) under Army Research Office (ARO) Contract No. DAAD19-01-1-0667.

\section{APPENDIX A: OVERLAP INTEGRALS}

In this appendix, we list the overlap integrals, as involved in the dynamic Eq. (12). Using the phase conventions as introduced in Eqs. (14) and (19), we find explicitly

$$
\begin{gathered}
J_{j}=n_{j}, \\
I_{j k}=n_{j} n_{k} \mathcal{O}_{\phi \theta}, \\
\left(\phi_{0} \theta_{0} \mid \phi_{-} \theta_{+}\right)=\left(\phi_{0} \theta_{0} \mid \phi_{+} \theta_{-}\right) \\
=\left(\phi_{0}^{2} \mid \theta_{+} \theta_{-}\right) \\
=n_{0}^{2} \mathcal{O}_{\phi \theta} / 2=2 n_{+} n_{-} \mathcal{O}_{\phi \theta}, \\
\left(\phi_{0}^{2} \mid \phi_{+} \phi_{-}\right)=n_{0}^{2} \mathcal{O}_{\phi \phi} / 2 .
\end{gathered}
$$

\section{APPENDIX B: THE ZERO MODE HAMILTONIAN}

The zero mode Hamiltonian is listed below.

$$
\begin{aligned}
\frac{H_{\text {zero }}}{N}= & -\frac{P_{+}^{2}}{J_{+}^{2}}\left[-\frac{J_{+}}{2} \tilde{u}+2 c_{-} I_{-+}+2 c_{+} I_{+0}+2 c_{2}\left(\phi_{0} \theta_{0} \mid \phi_{-} \theta_{+}\right)^{\prime}+c_{2}\left(\phi_{0}^{2} \mid \theta_{+} \theta_{-}\right)^{\prime}\right]-\frac{2 P_{0}^{2}\left[-\frac{J_{0}}{4} \tilde{u}+c_{+} I_{+0}+c_{+} I_{-0}\right.}{J_{0}} \\
& \left.+c_{2}\left(\phi_{-} \theta_{+} \mid \phi_{0} \theta_{0}\right)^{\prime}+c_{2}\left(\phi_{+} \theta_{-} \mid \phi_{0} \theta_{0}\right)^{\prime}\right]-\frac{P_{-}^{2}}{J_{-}^{2}}\left[-\frac{J_{-}}{2} \tilde{u}+2 c_{-} I_{-+}+2 c_{+} I_{-0}+2 c_{2}\left(\phi_{0} \theta_{0} \mid \phi_{+} \theta_{-}\right)^{\prime}+c_{2}\left(\phi_{0}^{2} \mid \theta_{+} \theta_{-}\right)^{\prime}\right] \\
& +\frac{4 P_{+} P_{0}}{J_{+} J_{0}}\left[c_{+} I_{+0}+c_{2}\left(\phi_{0} \theta_{0} \mid \phi_{-} \theta_{+}\right)^{\prime}\right]+\frac{4 P_{-} P_{0}}{J_{-} J_{0}}\left[c_{+} I_{-0}+c_{2}\left(\phi_{0} \theta_{0} \mid \phi_{+} \theta_{-}\right)^{\prime}\right]+\frac{2 P_{+} P_{-}}{J_{+} J_{-}}\left[2 c_{-} I_{-+}+c_{2}\left(\phi_{0}^{2} \mid \theta_{+} \theta_{-}\right)^{\prime}\right] \\
& -\frac{c_{2}}{\hbar^{2}}\left(\phi_{0}^{2} \mid \phi_{+} \phi_{-}\right)^{\prime}\left(\frac{Q_{+}}{J_{+}}-\frac{2 Q_{0}}{J_{0}}+\frac{Q_{-}}{J_{-}}\right)^{2} .
\end{aligned}
$$

\section{APPENDIX C: COEFFICIENTS IN THE ZERO MODE HAMILTONIAN FOR FERROMAGNETIC INTERACTIONS}

The coefficients in the zero mode Hamiltonian (24) are

$$
\begin{gathered}
a=\frac{1}{4 n_{0}^{2}}\left[\frac{\varepsilon}{3}\left(5+3 m^{2}\right)+2 \mathcal{O}_{\phi \theta}\left(3 c_{0}\left(1-m^{2}\right)^{2}+\frac{8}{3} c_{2}\left(1-3 m^{2}\right)\right)\right], \quad b=\frac{1}{2 n_{0}^{2}}\left[\varepsilon\left(1+m^{2}\right)-8 c_{2} \mathcal{O}_{\phi \theta} m^{2}\right], \\
c=\frac{1}{3 n_{0}^{2}}\left(\varepsilon-4 c_{2} \mathcal{O}_{\phi \theta}\right), \quad \alpha=\frac{\sqrt{2}}{6 n_{0}^{2}}\left(1+3 m^{2}\right)\left(\varepsilon-4 c_{2} \mathcal{O}_{\phi \theta}\right), \quad \beta=-\frac{2 m}{\sqrt{3} n_{0}^{2}}\left(\varepsilon-4 c_{2} \mathcal{O}_{\phi \theta}\right), \\
\gamma=-\frac{2 m}{n_{0}^{2}} \sqrt{\frac{2}{3}}\left[\varepsilon-c_{2} \mathcal{O}_{\phi \theta}\left(1+3 m^{2}\right)\right], \quad \eta=-3 c_{2} n_{0}^{2} \mathcal{O}_{\phi \phi} / \hbar^{2} .
\end{gathered}
$$

\section{APPENDIX D: PARAMETERS IN EVOLUTION MATRIX}

The list of parameters in evolution matrix $\mathcal{T}(t)$, of Eq. (33) are

$$
\begin{gathered}
\alpha^{\prime}=\frac{\alpha}{2 c}=\frac{1+3 m^{2}}{2 \sqrt{2}}, \quad \beta^{\prime}=\frac{\beta}{2 c}=-\sqrt{3} m, \quad \zeta_{a}=\frac{4 a c-\alpha^{2}}{2 c}=\frac{3\left(1+m^{2}\right) \varepsilon}{1-m^{2}}+12\left(c_{0}+c_{2}\right) \mathcal{O}_{\phi \theta}, \\
\zeta_{b}=\frac{4 b c-\beta^{2}}{2 c}=\frac{4 \varepsilon}{1-m^{2}}, \quad \zeta_{c}=\frac{2 c \gamma-\alpha \beta}{2 c}=-\frac{2 \sqrt{6} m \varepsilon}{1-m^{2}} .
\end{gathered}
$$




\section{APPENDIX E: EXPLICIT FORM OF NUMBER AND PHASE FLUCTUATION}

Here we present the explicit form of the solutions for the number and phase fluctuations.

$$
\begin{aligned}
& p_{N}(t)=p_{N}(0), \quad p_{M}(t)=p_{M}(0), \\
& p_{Y}(t)=-\frac{1+3 m^{2}}{2 \sqrt{2}}(1-\cos \Omega t) p_{N}(0)+\sqrt{3} m(1-\cos \Omega t) p_{M}(0)+p_{Y}(0) \cos \Omega t-\frac{q_{Y}(0)}{\hbar \Delta_{Y 0}^{2}} \sin \Omega t, \\
& q_{N}(t)=\left[N t\left(\frac{3\left(1+m^{2}\right) \varepsilon}{1-m^{2}}+12\left(c_{0}+c_{2}\right) \mathcal{O}_{\phi \theta}\right)+\frac{\left(1+3 m^{2}\right)^{2}}{8} \hbar \Delta_{Y 0}^{2} \sin \Omega t\right] p_{N}(0)-\left[N t \frac{2 \sqrt{6} m \varepsilon}{1-m^{2}}\right. \\
& \left.+\frac{\sqrt{3} m\left(1+3 m^{2}\right)}{2 \sqrt{2}} \hbar \Delta_{Y 0}^{2} \sin \Omega t\right] p_{M}(0)+\frac{1+3 m^{2}}{2 \sqrt{2}} \hbar^{2} \Delta_{Y 0}^{2} p_{Y}(0) \sin \Omega t+q_{N}(0)-\frac{1+3 m^{2}}{2 \sqrt{2}}(1-\cos \Omega t) q_{Y}(0), \\
& q_{M}(t)=-\left[N t \frac{2 \sqrt{6} m \varepsilon}{1-m^{2}}+\hbar \Delta_{Y 0}^{2} \frac{\sqrt{3} m\left(1+3 m^{2}\right)}{2 \sqrt{2}} \sin \Omega t\right] p_{N}(0)+\left(N t \frac{4 \varepsilon}{1-m^{2}}+3 m^{2} \hbar \Delta_{Y 0}^{2} \sin \Omega t\right) p_{M}(0) \\
& -\sqrt{3} m \hbar \Delta_{Y 0}^{2} p_{Y}(0) \sin \Omega t+q_{M}(0)+\sqrt{3} m(1-\cos \Omega t) q_{Y}(0), \\
& q_{Y}(t)=\hbar \Delta_{Y 0}^{2} \frac{1+3 m^{2}}{2 \sqrt{2}} p_{N}(0) \sin \Omega t-\hbar \Delta_{Y 0}^{2} \sqrt{3} m p_{M}(0) \sin \Omega t+\hbar \Delta_{Y 0}^{2} p_{Y}(0) \sin \Omega t+q_{Y}(0) \cos \Omega t, \\
& p_{+}^{\prime}(t)=\frac{3\left(1-m^{2}\right)+\left(1+3 m^{2}\right) \cos \Omega t}{4 \sqrt{3}} p_{N}(0)+\frac{1+m-m \cos \Omega t}{\sqrt{2}} p_{M}(0)+\frac{\cos \Omega t}{\sqrt{6}} p_{Y}(0)-\frac{\sin \Omega t}{\sqrt{6} \hbar \Delta_{Y 0}^{2}} q_{Y}(0) \\
& p_{0}^{\prime}(t)=\frac{3\left(1+m^{2}\right)-\left(1+3 m^{2}\right) \cos \Omega t}{2 \sqrt{3}} p_{N}(0)-\sqrt{2} m(1-\cos \Omega t) p_{M}(0)-\frac{2 \cos \Omega t}{\sqrt{6}} p_{Y}(0)+\frac{2 \sin \Omega t}{\sqrt{6} \hbar \Delta_{Y 0}^{2}} q_{Y}(0) \\
& p_{-}^{\prime}(t)=\frac{3\left(1-m^{2}\right)+\left(1+3 m^{2}\right) \cos \Omega t}{4 \sqrt{3}} p_{N}(0)-\frac{1-m+m \cos \Omega t}{\sqrt{2}} p_{M}(0)+\frac{\cos \Omega t}{\sqrt{6}} p_{Y}(0)-\frac{\sin \Omega t}{\sqrt{6} \hbar \Delta_{Y 0}^{2}} q_{Y}(0) \\
& q_{+}^{\prime}(t)=\left[\sqrt{3} N t\left(\frac{(1-m) \varepsilon}{1+m}+4\left(c_{0}+c_{2}\right) \mathcal{O}_{\phi \theta}\right)+\frac{\sqrt{3} \hbar \Delta_{Y 0}^{2}}{8}\left(1+3 m^{2}\right)(1+m)^{2} \sin \Omega t\right] p_{N}(0) \\
& +\left[\frac{2 \sqrt{2} \varepsilon}{1+m} N t-\frac{3 m(1-m)^{2}}{2 \sqrt{2}} \hbar \Delta_{Y 0}^{2} \sin \Omega t\right] p_{M}(0)+\hbar \Delta_{Y 0}^{2} \frac{3(1-m)^{2} \sin \Omega t}{2 \sqrt{6}} p_{Y}(0) \\
& +\frac{1}{\sqrt{3}} q_{N}(0)+\frac{1}{\sqrt{2}} q_{M}(0)+\left[-\frac{1-6 m+3 m^{2}}{2 \sqrt{6}}+\frac{(1-m)^{2}}{2 \sqrt{2}} \cos \Omega t\right] q_{Y}(0), \\
& q_{0}^{\prime}(t)=\left[\sqrt{3} N t\left(\frac{1+m^{2}}{1-m^{2}} \varepsilon+4\left(c_{0}+c_{2}\right) \mathcal{O}_{\phi \theta}\right)+\frac{\sqrt{3}}{8} \hbar \Delta_{Y 0}^{2}\left(3 m^{4}-2 m^{2}-1\right) \sin \Omega t\right] p_{N}(0)+\left[-\frac{2 \sqrt{2} m \varepsilon}{1-m^{2}} N t\right. \\
& \left.+\hbar \Delta_{Y 0}^{2} \frac{3 m\left(1-m^{2}\right)}{2 \sqrt{2}} \sin \Omega t\right] p_{M}(0)+\hbar \Delta_{Y 0}^{2} \frac{3 m^{2}-3}{2 \sqrt{6}} \sin \Omega p_{Y}(0)+\frac{1}{\sqrt{3}} q_{N}(0)+\left[-\frac{1+3 m^{2}}{2 \sqrt{6}}+\frac{3 m^{2}-3}{2 \sqrt{6}} \cos \Omega t\right] q_{Y}(0)
\end{aligned}
$$




$$
\begin{aligned}
q_{-}^{\prime}(t)= & {\left[\sqrt{3} N t\left(\frac{(1+m) \varepsilon}{1-m}+4\left(c_{0}+c_{2}\right) \mathcal{O}_{\phi \theta}\right)+\frac{\sqrt{3} \hbar \Delta_{Y 0}^{2}}{8}\left(1+3 m^{2}\right)\left(1+m^{2}\right) \sin \Omega t\right] p_{N}(0) } \\
& -\left[\frac{2 \sqrt{2} \varepsilon}{1-m} N t+\frac{3 m(1+m)^{2}}{2 \sqrt{2}} \hbar \Delta_{Y 0}^{2} \sin \Omega t\right] p_{M}(0)+\hbar \Delta_{Y 0}^{2} \frac{3(1+m)^{2} \sin \Omega t}{2 \sqrt{6}} p_{Y}(0) \\
& +\frac{1}{\sqrt{3}} q_{N}(0)-\frac{1}{\sqrt{2}} q_{M}(0)+\left[-\frac{1+6 m+3 m^{2}}{2 \sqrt{6}}+\frac{(1+m)^{2}}{2 \sqrt{2}} \cos \Omega t\right] q_{Y}(0)
\end{aligned}
$$

\section{APPENDIX F: THE VARIANCE OF MACROSCOPIC CONDENSATE SPIN IN CARTESIAN COORDINATES}

In Cartesian coordinates, the variances of the condensate spin direction is given by

$$
\begin{gathered}
\left\langle\left[\delta f_{x}(t)\right]^{2}\right\rangle=\frac{1}{1-m^{2}}\left[3\left\langle p_{N}^{2}(0)\right\rangle-2 \sqrt{6} m\left\langle p_{N}(0) p_{M}(0)\right\rangle+2 m^{2}\left\langle p_{M}^{2}(0)\right\rangle\right], \\
\left\langle\left[\delta f_{y}(t)\right]^{2}\right\rangle=\frac{1-m^{2}}{2 \hbar^{2}}\left[\left\langle q_{M}^{2}(0)+2 \sqrt{3} m\left\langle q_{M}(0) q_{Y}(0)\right\rangle+3 m^{2}\left\langle q_{Y}^{2}(0)\right\rangle\right]+\frac{4 N^{2} \varepsilon^{2} t^{2}}{\hbar^{2}\left(1-m^{2}\right)}\left[3 m^{2}\left\langle p_{N}^{2}(0)\right\rangle-2 \sqrt{6} m\left\langle p_{N}(0) p_{M}(0)\right\rangle\right.\right. \\
\left.+2\left\langle p_{M}^{2}(0)\right\rangle\right]+\frac{\sqrt{2} N \varepsilon t}{\hbar^{2}}\left\{\sqrt{2}\left[\left\langle q_{M}(0) p_{M}(0)\right\rangle+\left\langle p_{M}(0) q_{M}(0)\right\rangle\right]-\sqrt{3} m\left[\left\langle q_{M}(0) p_{N}(0)\right\rangle+\left\langle p_{N}(0) q_{M}(0)\right\rangle\right]\right. \\
\left.+\sqrt{6} m\left[\left\langle q_{Y}(0) p_{N}(0)\right\rangle+\left\langle p_{N}(0) q_{Y}(0)\right\rangle\right]-3 m^{2}\left[\left\langle q_{Y}(0) p_{M}(0)\right\rangle+\left\langle p_{M}(0) q_{Y}(0)\right\rangle\right]\right\} \\
\left\langle\left[\delta f_{z}(t)\right]^{2}\right\rangle=2\left\langle p_{M}^{2}(0)\right\rangle .
\end{gathered}
$$

\section{APPENDIX G: COEFFICIENTS IN THE ZERO MODE HAMILTONIAN FOR ANTIFERROMAGNETIC INTERACTIONS}

The coefficients of zero mode Hamiltonian (77) for antiferromagnetic interactions (with $\mathcal{M}=0$ )

$$
\begin{gathered}
a=\frac{\left(1+3 n_{0}\right) \varepsilon}{6 n_{0}\left(1-n_{0}\right)}+\left[6 c_{0}-\frac{8 c_{2} n_{0}}{3\left(1-n_{0}\right)}\right] \mathcal{O}_{\phi \theta}, \quad b=\frac{\varepsilon}{1-n_{0}}+4 c_{2} \mathcal{O}_{\phi \theta}, \quad c=\frac{\varepsilon}{3 n_{0}\left(1-n_{0}\right)}+\frac{4 c_{2} n_{0}}{3\left(1-n_{0}\right)} \mathcal{O}_{\phi \theta}, \\
\alpha=\frac{2\left(3 n_{0}-1\right) \varepsilon}{3 \sqrt{2} n_{0}\left(1-n_{0}\right)}+\frac{16 c_{2} n_{0}}{3 \sqrt{2}\left(1-n_{0}\right)} \mathcal{O}_{\phi \theta}, \quad \eta=3 c_{2} n_{0}\left(1-n_{0}\right) \mathcal{O}_{\phi \phi} / \hbar^{2} .
\end{gathered}
$$

[1] M.H. Anderson, J.R. Ensher, M.R. Matthews, C.E. Wieman, and E.A. Cornell, Science 269, 198 (1995).

[2] K.B. Davis, M.-O. Mewes, M.R. Andrews, N.J. van Druten, D.S. Durfee, D.M. Kurn, and W. Ketterle, Phys. Rev. Lett. 75, 3969 (1995).

[3] C.C. Bradley, C.A. Sackett, J.J. Tollett, and R.G. Hulet, Phys. Rev. Lett. 75, 1687 (1995); ibid. 79, 1170 (1997).

[4] M. Lewenstein and L. You, Phys. Rev. Lett. 77, 3489 (1996).

[5] E.M. Wright, D.F. Walls, and J.C. Garrison, Phys. Rev. Lett. 77, 2158 (1996).

[6] A. Imamoglu, M. Lewenstein, and L. You, Phys. Rev. Lett. 78, 2511 (1997).

[7] P. Villain, M. Lewenstein, R. Dum, Y. Castin, L. You, A. Imamoglu, and T.A.B. Kennedy, J. Mod. Opt. 44, 1775 (1997).

[8] C.K. Law, H. Pu, N.P. Bigelow, and J.H. Eberly, Phys. Rev. A 58, 531 (1998).

[9] P. Villain and M. Lewenstein, Phys. Rev. A 59, 2250 (1999).
[10] M.R. Andrews, C.G. Townsend, H.-J. Miesner, D.S. Durfee, D.M. Kurn, and W. Ketterle, Science 275, 637 (1997).

[11] C. Orzel, A.K. Tuchman, M.L. Fenselau, M. Yasuda, and M.A. Kasevich, Science 291, 2386 (2001).

[12] M. Greiner, O. Mandel, T.W. Hansch, and I. Bloch, Nature (London) 419, 51 (2002).

[13] D. Jaksch, C. Bruder, J.I. Cirac, C.W. Gardiner, and P. Zoller, Phys. Rev. Lett. 81, 3108 (1998).

[14] D.M. Stemper-Kurn, M.R. Andrews, A.P. Chikkatur, S. Inouye, H.-J. Miesner, J. Stenger, and W. Ketterle, Phys. Rev. Lett. 80, 2027 (1998).

[15] M. Barrett, J. Sauer, and M.S. Chapman, Phys. Rev. Lett. 87, 010404 (2001).

[16] J.A. Dunningham and K. Burnett, Phys. Rev. Lett. 82, 3729 (1999).

[17] J. Javanainen, J. Phys. B 33, 5493 (2000); S. Ashhab and A.J. Leggett, Phys. Rev. A 65, 023604 (2002). 
[18] S. Yi, Ö.E. Müstecaplıoğlu, and L. You, Phys. Rev. Lett. 90, 140404 (2003).

[19] T.-L. Ho, Phys. Rev. Lett. 81, 742 (1998).

[20] S. Yi, Ö.E. Müstecaplıoğlu, Chang-Pu Sun, and L. You, Phys. Rev. A 66, 011601(R) (2002).

[21] P. Nozieres and D. Pines, The Theory of Quantum Liquids (Addison-Wesley, Reading, MA, 1990), Vol. 2.

[22] T. Ohmi and K. Machida, J. Phys. Soc. Jpn. 67, 1822 (1998).

[23] E.V. Goldstein and P. Meystre, Phys. Rev. A 59, 3896 (1998).

[24] H. Pu, C.K. Law, and N.P. Bigelow, Physica B 280, 27 (2000); C.K. Law, H. Pu, and N.P. Bigelow, Phys. Rev. Lett. 81, 5257 (1998).
[25] T. Isoshima, K. Machida, and T. Ohmi, Phys. Rev. A 60, 4857 (1999).

[26] N.P. Robins, W. Zhang, E.A. Ostrovskaya, and Y.S. Kivshar, Phys. Rev. A 64, 021601(R) (2001).

[27] E.G.M. van Kempen, S.J.J.M.F. Kokkelmans, D.J. Heinzen, and B.J. Verhaar, Phys. Rev. Lett. 88, 093201 (2002).

[28] Ö. Müstecaplıoğlu, M. Zhang, and L. You, Phys. Rev. A 66, 033611 (2002).

[29] F. Dalfovo, L. Pitaevskii, and S. Stringari, Phys. Rev. A 54, 4213 (1996).

[30] T.-L. Ho and S.K. Yip, Phys. Rev. Lett. 84, 4031 (2000).

[31] M. Koashi and M. Ueda, Phys. Rev. Lett. 84, 1066 (2000). 